pag

Business School

WORKING PAPER SERIES

Working Paper

2014-336
Optimal Portfolio Positioning within Generalized Johnson Distributions

Naceur Naguez

Jean-Luc Prigent

http://www.ipag.fr/fr/accueil/la-recherche/publications-WP.html

IPAG Business School

184, Boulevard Saint-Germain

75006 Paris

France 


\title{
Optimal Portfolio Positioning within Generalized Johnson Distributions
}

\author{
Naceur Naguez* Jean-Luc Prigent ${ }^{\dagger}$
}

This version: May 2014.

\begin{abstract}
Many empirical studies have shown that financial asset returns do not always exhibit Gaussian distributions. For example, it is well known that most hedge fund returns have significant skewness and kurtosis, which are far from being those of a normal distribution. The introduction of the family of Johnson distributions allows to overcome the problem of fitting empirical financial data, since it contains a large class of probability distributions. Additionally, this class can be extended to a quite general family of distributions by considering all possible regular transformations of the standard Gaussian law. In this framework, we consider the portfolio optimal positioning problem, which has been first addressed by Brennan and Solanki (1981) and by Leland (1980). We assume that the investor wants to maximize the expected utility of his portfolio value, which corresponds to a given function of some specific portfolio of common risky assets. Such problem has been previously examined for Gaussian log returns (see Carr and Madan, 2001; Prigent, 2006). We determine and analyze the optimal portfolio for log return having Johnson distributions. As a by-product, we introduce the notion of Johnson stochastic processes. The solution is characterized for arbitrary utility functions and illustrated in particular for a CRRA utility. Our findings show how the profiles of financial structured products must be selected when taking account of non Gaussian log-returns.
\end{abstract}

\footnotetext{
*University of Cergy-Pontoise, THEMA. 33 Bd du Port, 95011, Cergy-Pontoise, France. E-mail: naceur.naguez@u-cergy.fr

${ }^{\dagger}$ IPAG Business School and University of Cergy-Pontoise, THEMA. 33 Bd du Port, 95011, Cergy-Pontoise, France. E-mail: jean-luc.prigent@u-cergy.fr
} 


\section{Introduction}

Most of studies dealing with the optimality of financial portfolio assume that the investor searches for maximizing the expected utility of his final portfolio value. Usually, these models are developed in continuous time (see Cox and Huang, 1989; Cvitanic and Karatzas, 1996). Portfolio insurance problems are also examined in such a framework (see Grossman and Vila,1989; Basak, 1995; Grossman and Zhou 1996...). El Karoui et al. (2001) show that, for a fixed guarantee at maturity, the OBPI ("Option Based Portfolio Strategy") type methods are optimal for general utility functions (see also Jensen and Sorensen (2001) for a particular case). Another stream of literature has focused on the problem of static optimal positioning. This latter one has been first addressed in the context of partial equilibrium by Brennan and Solanki (1981) and by Leland (1980). The management holds in one-period setting. The portfolio value is a function of a reference portfolio or asset ("a benchmark"). The optimal payoff is deduced from the maximization of investor's expected utility. The optimal portfolio profile depends crucially on the individual's risk aversion. Following this approach, Carr and Madan (2001) consider a financial market in which all types of European options (calls and puts with all possible strikes) are available. This last assumption implies that to the market is (statically) complete, which is the counterpart of the dynamic completeness in continuous time. Such hypothesis can be justified when there is a sufficient number of options available on the financial market (the S\&P 500, the CAC 40 for example). Note that discrete time rebalancing assumption is more in accordance with usual practice. This is particularly due to transaction costs and liquidity problems that may be encountered during the sale or purchase of financial assets.

Among various portfolio strategies, portfolio insurance methods have been specifically introduced first to allow the investor to recover at maturity a predefined proportion of his initial investment, whatever the future evolution of financial markets, second to provide significant benefits from potential market rises. Usually, the portfolio payoff depends on the value of a reference portfolio (a financial stock index for instance). The two main portfolio insurance methods are the OBPI (Option Based Portfolio Insurance) and the CPPI (Constant Proportion Portfolio Insurance). The OBPI has been introduced by Leland and Rubinstein (1976). It is based on an investment on a risky asset $S$ (usually a financial index such as $S \& P 500$ ) covered by the simultaneous purchase of a put on that asset. This combination allows to provide the guarantee since, whatever the value of $S$ at maturity $T$, the portfolio value is always above the strike price $K$ of the put. The CPPI method ${ }^{1}$ has been introduced by Perold (1986) and further extended by Perold and Sharpe (1988) for fixed income investments

\footnotetext{
${ }^{1}$ This method is based on a relatively simple strategy to dynamically allocate the portfolio. The investor begins by determining a floor equal to the smallest acceptable portfolio value (discounted), depending on his desired guarantee at maturity. Then he defines the cushion as the difference in excess of the value of the portfolio and the floor. The amount invested in the risky asset is called exposure. It corresponds to the value of this cushion multiplied by a predetermined factor, called the "multiple". This leads to the fact that when the value of
} 
and Black and Jones (1987) for equity investments. ${ }^{2}$ Consequently, OBPI is an extension of the optimal positioning problem, by taking additional guarantee constraints into account.

Standard analysis of optimal portfolio positioning is based on the normality assumption for log-returns. However, such hypothesis is rather restrictive since many financial assets exhibit clearly non-Gaussian log-returns. For example, the empirical analysis of hedge fund returns has shown that the mean-variance approach is not adequate to study the risk and performance of these funds. Using data with monthly frequency, Fung and Hsieh (1997), Brooks and Kat (2002), Amenc et al. (2003) demonstrate that distributions of hedge funds returns have a negative skewness and an excess kurtosis. In order to better estimate the returns having these statistical characteristics, it is necessary to take higher order moments into account. The Johnson system that is a system that covers different families of distribution, can take into account the four first moments of distribution. As mentioned in Passow (2004), this family of probability distributions can calibrate independently the four first moments, "while disregarding potentially insignificant moments of order higher than 5." The introduction of Johnson distributions allows to cover up to fourth-order moment patterns of (Log-) Normal, (2-sided) Student t- and Weibull distributions. The family of Edgeworth expansions and twelve different types of Pearson distributions are also included. Note that these latter distributions include 1st and 2nd kind Beta and Gamma distributions. This is the reason why Johnson distributions can actually model the specific moment patterns.

In this paper, our goal is to introduce Johnson distributions (describing the benchmark log-return) and to examine their impact on the portfolio optimal positioning and portfolio insurance strategies such as the OBPI and its extensions. ${ }^{3}$ Thus, we extend previous results of Carr and Madan (2001) for optimal positioning and of Bertrand, Lesne and Prigent (2001), Prigent (2006) devoted specifically to portfolio insurance, in particular to the generalization of the OBPI method. This paper is organized as follows. Section 2 is devoted to Johnson

the cushion approaches zero, exposure approaches zero as well. In continuous time and in the absence of jumps in the dynamics of the risky asset reference, this method allows to maintain the portfolio value above the floor, which is exactly the purpose of the guarantee.

${ }^{2}$ The comparison between the OBPI and the CPPI was conducted using various criteria Black and Rouhani (1989), Black and Perold (1992), Bookstaber and Langsam (2000), Bertrand and Prigent (2001). The hypothesis of lognormal returns is generally used in these studies (see Bertrand and Prigent (2006) and Prigent (2007) for more details on the portfolio insurance and in particular on the comparison).

${ }^{3}$ In this context, we can illustrate how the profile of the optimal portfolio (maximizing expected utility) depends crucially on the risk aversion of the investor, and his prudence (see Eeckhoudt and Gollier, 2005, Bertrand and Prigent, 2010). By assuming that there are European options puts and calls with all the desired exercise price, the financial market is theoretically complete. In practice, it is best to reconstruct the determined optimum profile with the available options on the market. This approximation is justified when there are many options on the underlying (for example, the S \& P500). This hypothesis is the counterparty for the completeness of the market in continuous time, obtained through the dynamic hedging of options. 
distributions and its generalization. We also introduce the notion of Johnson processes to model continuous-time dynamics of risky assets. We provide main mathematical properties of such a class of stochastic processes. In section 3 we determine the optimal portfolio payoff when the risky asset is assumed to follow the generalized Johnson distribution. A fundamental example is detailed, particularly with respect to maximizing the expected utility of CRRA type. We compare the optimal solution to that of the optimal positioning in the standard geometric Brownian case. Finally, Section 4 contains the concluding remarks. Some of the proofs are gathered in the Appendix.

\section{Johnson distributions and Johnson processes}

In what follows, we deal with the optimal positioning problem in the presence of Johnson probability distributions. For this purpose, we begin by recalling definitions and main properties of Johnson distributions. Then, we introduce the notion of Johnson stochastic processes. This is an extension of the standard Johnson distributions to the continuous time case. Finally, we provide the general solution of the optimal positioning in financial derivatives within Johnson stochastic processes.

\subsection{Johnson distributions}

The Johnson system covers three main families of distributions that can model a wide variety of empirical distributions. Given a continuous random variable $X, X$ has a Johnson distribution if there exists a non decreasing and continuous function $g$ such that:

$$
Z=\gamma+\delta g\left(\frac{X-\xi}{\lambda}\right)
$$

where $Z$ is a standard normal random variable, and $\gamma, \delta, \xi, \lambda$ are given parameters. Usually, $\gamma, \delta$ correspond to trend parameters, $\xi$ is a positional parameter and finally $\lambda$ corresponds to a scaling parameter.

Notation 1 We introduce the function $l$ and its inverse by setting:

$$
\begin{aligned}
l(x) & =\gamma+\delta g\left(\frac{x-\xi}{\lambda}\right), \\
l^{-1}(z) & =\lambda g^{-1}\left(\frac{z-\gamma}{\delta}\right)+\xi .
\end{aligned}
$$

Denote also $y=\left(\frac{x-\xi}{\lambda}\right)$.

Function $g($.$) is usually one of the three following functions:$

- For the Lognormal distribution:

$$
S_{L}: g(y)=\ln (y) .
$$


- For the unbounded distribution:

$$
S_{U}: g(y)=\ln \left(y+\sqrt{1+y^{2}}\right)=\sinh ^{-1}(y) .
$$

- For the bounded distribution:

$$
S_{B}: g(y)=\ln \left(\frac{y}{1-y}\right) .
$$

The $S_{U}$ distribution is unbounded in both directions while the $S_{B}$ distribution is bounded in both directions $(\xi, \xi+\lambda)$. The support $\Omega$ of each distribution is as follows:

$$
\Omega=\left\{\begin{array}{c}
\left.S_{L}:\right] \xi,+\infty[ \\
\left.S_{U}:\right]-\infty,+\infty[ \\
\left.S_{B}:\right] \xi, \xi+\lambda[
\end{array}\right.
$$

The Johnson probability density function (pdf) $f_{X}$ is easily deduced from the standard normal distribution. It is given by canonical transformations of the standard Gaussian distribution:

$$
\begin{gathered}
f_{X}(x)= \\
\frac{\delta}{\lambda \sqrt{2 \pi}} \exp \left[-\frac{1}{2}\left\{\gamma+\delta g\left(\frac{x-\xi}{\lambda}\right)\right\}^{2}\right]\left|g^{\prime}\left(\frac{x-\xi}{\lambda}\right)\right| .
\end{gathered}
$$

The pdf $f_{X}$ corresponding to each of the three main Johnson distributions is also easily deduced from previous general form:

$$
\begin{gathered}
S_{L}: f_{X}(x)= \\
\frac{\delta}{\lambda \sqrt{2 \pi}\left(\frac{x-\xi}{\lambda}\right)} \exp \left[-\frac{1}{2}\left\{\gamma+\delta \ln \left(\frac{x-\xi}{\lambda}\right)\right\}^{2}\right] \text { for } \frac{x-\xi}{\lambda}<0 . \\
\frac{\delta}{\lambda \sqrt{2 \pi} \sqrt{\left(\frac{x-\xi}{\lambda}\right)^{2}+1}} \exp \left[-\frac{1}{2}\left\{\gamma+\delta \ln \left(\frac{x-\xi}{\lambda}+\sqrt{\left(\frac{x-\xi}{\lambda}\right)^{2}+1}\right)\right\}^{2}\right] . \\
\quad S_{B}: f_{X}(x)= \\
\text { for } 0<\frac{x-\xi}{\lambda}<1 .
\end{gathered}
$$


Proposition 2 The general form of the cdf $F_{X}$ of $X$ is given by:

$$
F_{X}(x)=\Phi\left(\gamma+\delta g\left(\frac{x-\xi}{\lambda}\right)\right)
$$

with $\Phi(x)=\frac{1}{\sqrt{2 \pi}} \int_{-\infty}^{x} \exp \left(-\frac{t^{2}}{2}\right) d t$.

The pdf is given by:

$$
f_{X}(x)=\Phi^{\prime}\left(\gamma+\delta g\left(\frac{x-\xi}{\lambda}\right)\right) \frac{\delta}{\lambda} g^{\prime}\left(\frac{x-\xi}{\lambda}\right) .
$$

When using Johnson distributions, we can for example search for one of the main Johnson distributions. Therefore, in that case, the first step is to determine which of the three families should be used. The second step is to estimate the parameters of this distribution. ${ }^{4}$ We can also determine the best function $g$, using a non parametric approach. In that latter case, the function $g$ may no longer belong to one of the three basic families.

\subsection{Johnson stochastic processes}

We suppose that the risky asset $S_{t}$ is given at any time by:

$$
S_{t}=S_{0} \exp \left[X_{t}\right],
$$

where $X$ denotes the process which is equal to the standard Brownian motion $W$ transformed through the relation:

$$
X_{t}=\lambda_{t} g^{-1}\left(\frac{W_{t}-\gamma_{t}}{\delta_{t}}\right)+\xi_{t}
$$

which is equivalent to:

$$
W_{t}=\gamma_{t}+\delta_{t} g\left(\frac{X_{t}-\xi_{t}}{\lambda_{t}}\right)
$$

where $\gamma_{t}, \delta_{t}, \xi_{t}, \lambda_{t}$ are deterministic and differentiable functions and $g$ is a fixed function which is twice differentiable. Recall that the function $l_{t}$ and its inverse $l_{t}^{-1}$ are defined by:

$$
\begin{aligned}
l_{t}(x) & =\gamma_{t}+\delta_{t} g\left(\frac{x-\xi_{t}}{\lambda_{t}}\right), \\
l_{t}^{-1}(z) & =\lambda_{t} g^{-1}\left(\frac{z-\gamma_{t}}{\delta_{t}}\right)+\xi_{t} .
\end{aligned}
$$

The information is modeled through the filtration defined by: $\mathcal{F}_{t}=\sigma\left(W_{s}\right)_{s \leq t}$, where $\mathcal{F}_{0}$ is the augmented $\sigma$-algebra of the standard Brownian motion. Note that $\mathcal{F}_{0^{+}}=\mathcal{F}_{0}$. The notations and definitions are those of Jacod and Shiryaev (2003). All processes are defined on a filtered probability space $\left(\Omega, \mathcal{F},\left(\mathcal{F}_{t}\right), \mathbb{P}\right)$ indexed on $[0, T]$ and satisfying the usual conditions.

\footnotetext{
${ }^{4}$ Different methods can be used to estimate the Johnson parameters. The main ones are developed by Hill et al. (1976), Wheeler and Robert (1980), and Slifker and Shapiro (1980).
} 
Proposition 3 The conditional distributions of the Johnson process $X$ are also Johnson distributions.

Proof. A COMPLETER

Proposition 4 The Johnson process $X$ is a diffusion process, in particular a Markov process.

Proof. Using Ito's lemma and Yor's formula, the process $X$ is a solution of:

$$
\begin{aligned}
& d X_{t}=d\left[\lambda_{t} g^{-1}\left(\frac{W_{t}-\gamma_{t}}{\delta_{t}}\right)+\xi_{t}\right]=\lambda_{t}^{\prime} g^{-1}\left(\frac{W_{t}-\gamma_{t}}{\delta_{t}}\right) d t \\
& +\lambda_{t}\left(\left[g^{-1}\right]^{\prime}\left(\frac{W_{t}-\gamma_{t}}{\delta_{t}}\right)\left(-\frac{\delta_{t}^{\prime}}{\delta_{t}^{2}} W_{t}-\frac{\gamma_{t}^{\prime} \delta_{t}-\gamma_{t} \delta_{t}^{\prime}}{\delta_{t}^{2}}\right)\right) d t \\
& \quad+\frac{\lambda_{t}}{2}\left[g^{-1}\right] "\left(\frac{W_{t}-\gamma_{t}}{\delta_{t}}\right) \frac{1}{\delta_{t}^{2}} d t+\xi_{t}^{\prime} d t \\
& \quad+\lambda_{t}\left[g^{-1}\right]^{\prime}\left(\frac{W_{t}-\gamma_{t}}{\delta_{t}}\right) \frac{1}{\delta_{t}} d W_{t} .
\end{aligned}
$$

Assuming function $g$ is twice differentiable with non negative derivatives, then we have:

$$
\left[g^{-1}\right] \prime=\frac{1}{\left(g^{\prime} \circ g^{-1}\right)} \text { and }\left[g^{-1}\right]=-\frac{\left(g^{\prime} \circ g^{-1}\right)}{\left(g^{\prime} \circ g^{-1}\right)^{3}} \text {. }
$$

Thus, the process $X$ is a diffusion process, solution of the following stochastic differential equation (SDE):

$$
d X_{t}=a\left(t, X_{t}\right) d t+b\left(t, X_{t}\right) d W_{t},
$$

with

$$
\begin{aligned}
a(t, x)= & \lambda_{t}^{\prime}\left(\frac{x-\xi_{t}}{\lambda_{t}}\right)+\frac{\lambda_{t}}{g^{\prime}\left(\frac{x-\xi_{t}}{\lambda_{t}}\right)}\left(\left(-\frac{\delta_{t}^{\prime}}{\delta_{t}^{2}} l_{t}(x)-\frac{\gamma_{t}^{\prime} \delta_{t}-\gamma_{t} \delta_{t}^{\prime}}{\delta_{t}^{2}}\right)\right) \\
& -\frac{\lambda_{t}}{2 \delta_{t}^{2}} \frac{g^{\prime \prime}}{g^{\prime 3}}\left(\frac{x-\xi_{t}}{\lambda_{t}}\right)+\xi_{t}^{\prime} \\
b(t, x)= & \frac{\lambda_{t}}{\delta_{t} g^{\prime}\left(\frac{x-\xi_{t}}{\lambda_{t}}\right)} .
\end{aligned}
$$

Proposition 5 The Johnson process $X$ is a semimartingale with the following decomposition:

$$
X_{t}=A_{t}^{X}+M_{t}^{X},
$$

where $A_{t}^{X}$ and $M_{t}^{X}$ respectively denote the bounded variation component and the martingale part of the process $X$. They are defined from the following relations:

$$
d A_{t}^{X}=a\left(t, X_{t}\right) d t \text { and } d M_{t}^{X}=b\left(t, X_{t}\right) d W_{t} .
$$


Corollary 6 The predictable compensator $\langle X, X\rangle_{t}$ of process $X$ is given by:

$$
\langle X, X\rangle_{t}=\int_{0}^{t} b^{2}\left(s, X_{s}\right) d s .
$$

In what follows, the process of the discounted risky asset price is denoted by $\left(\widetilde{S}_{t}\right)_{t}$. It is a process with values in $\mathbb{R}$ which is a special semimartingale having the following canonical decomposition:

$$
\widetilde{S}=S_{0}+M+A,
$$

where $M$ is a local martingale and $A$ a predictable process with finite variation. We denote by $\langle M, M\rangle$ the predictable compensator of the martingale $M$.

Applying Ito's lemma, the risky asset $S_{t}$ follows the following dynamics:

$$
\frac{d S_{t}}{S_{t}}=d X_{t}+1 / 2 d\langle X, X\rangle_{t} .
$$

Remark 7 We can inverse the relationship between $S_{t}$ and $W_{t}$ since we have:

$$
X_{t}=\ln \left(\frac{S_{t}}{S_{0}}\right)
$$

which implies:

$$
W_{t}=\gamma_{t}+\delta_{t} g\left(\frac{\ln \left(\frac{S_{t}}{S_{0}}\right)-\xi_{t}}{\lambda_{t}}\right) .
$$

In what follows, we determine the risk-neutral probability of the financial market. From the relationship (8), $S$ is a solution of the following stochastic differential equation:

$$
d S_{t}=S_{t-} d R_{t}
$$

with

$$
d R_{t}=d X_{t}+1 / 2 d\langle X, X\rangle_{t} .
$$

Denote that $S$ is the stochastic exponential of Doléans-Dade of process $R$ : $S_{t}=S_{0} \mathcal{E}\left(R_{t}\right)$.

The bounded variation process $A_{t}^{R}$ and the martingale part $M_{t}^{R}$ of the process $R$ are respectively defined by:

$$
\begin{aligned}
d A_{t}^{R} & =d A_{t}^{X}+1 / 2 d\langle X, X\rangle_{t}, \\
d M_{t}^{R} & =d M_{t}^{X}=\frac{\lambda_{t}}{\delta_{t} g^{\prime}\left(\frac{x-\xi_{t}}{\lambda_{t}}\right)} d W_{t} .
\end{aligned}
$$




\section{Optimal positioning in the standard case}

In what follows, we first recall the standard results about the optimal portfolio positioning.

\subsection{Optimal portfolio: the case of dynamic completeness}

Suppose that the financial market is complete, without arbitrage opportunity and without friction. Prices of financial assets are assumed to be diffusion processes. Depending on the investor's risk aversion and management period, the portfolio manager chooses to allocate initial invested amount on different financial assets, in particular on a money market account $N$ with maturity $T$ indexed on the spot interest rate $r$. The portfolio resulting value $\left(V_{t}\right)_{t}$ is selffinancing. This means that the process $\left(V_{t} \exp (-r t)\right)_{t}$ is a $\mathbb{Q}$-martingale where $\mathbb{Q}$ is the risk-neutral probability.

We denote by $\eta=\frac{d \mathbb{Q}}{d \mathbb{P}}$ the Radon-Nikodym derivative of $\mathbb{Q}$ with respect to the historical probability $\mathbb{P}$. Denote by $M_{T}$ the process $\eta_{T} \exp (-r T)$. Due to the no-arbitrage condition, the budget constraint corresponds to the following relation:

$$
V_{0}=\mathbb{E}_{\mathbb{Q}}\left[V_{T} \exp (-r T)\right]=\mathbb{E}_{\mathbb{P}}\left[V_{T} M_{T}\right] .
$$

Suppose that the investor wants to maximize his expected utility under the historical probability $\mathbb{P}$. As usual, the utility $U$ of the investor is assumed to be increasing, concave and twice differentiable. Suppose as in Karatzas et al. (1986) that the marginal utility $U^{\prime}$ satisfies:

$$
\lim _{0+} U^{\prime}=+\infty \text { and } \lim _{+\infty} U^{\prime}=0 .
$$

Denote by $J$ the inverse of the marginal utility $U^{\prime}$.

The investor maximizes his expected utility:

$$
\left.\operatorname{Max} \mathbb{E}\left[U\left[V_{T}\right)\right]\right],
$$

where $V_{T}$ is the value of the portfolio payment at maturity $T$.

As proved in Cox and Huang (1989), the first order condition allows to determine the optimal portfolio:

$$
V_{T}^{*}=J\left[\rho \eta_{T}\right],
$$

where $J$ is the inverse of the marginal utility $U$ and $\rho$ is the Lagrange parameter associated to the budget constraint.

The proof is based on the fact that due to market completeness, there exists a one-to-on mapping between the set of all possible strategies and the set of all possible portfolio values at maturity. Therefore, these latter ones can be chosen as new variables for the optimization problem. Advantage of such transformation is that these variables are only submitted to a single constraint: the budget one. In addition, the budget constraint is a linear function of $V$. Therefore, 
there exists one and only one solution $V$ which is solution of $\frac{\partial L}{\partial V}=0$, where the Lagrangian $L$ is defined by:

$$
L(V, \lambda)=\int[U(V(s))] \mathbb{P}_{S_{T}}(d s)+\rho\left(V_{0}-\int v \eta_{T}(s) \mathbb{P}_{S_{T}}(d s)\right)
$$

and $\lambda$ is the Lagrange parameter associated to the budget constraint. Consequently, $V$ is the solution of $U^{\prime}(V)=\rho \eta_{T}$. Hence, we have:

$$
V_{T}^{*}=J\left[\lambda \eta_{T}\right] .
$$

\subsection{Optimal portfolio: static payment as function of the benchmark}

Suppose now that the investor must choose his whole portfolio strategy at the initial date ("buy-and-hold case"). Assume also that the final portfolio payoff is a function of the terminal value of the benchmark: $V_{T}=h\left(S_{T}\right)$. In this framework, the portfolio value can involve any derivative written on $S_{T}$. To illustrate the model, suppose that there exists two basic financial assets: the cash to which is associated the discount factor $N$, and the stock $S$ (a financial stock index, for example). We assume that the investor determines the optimal payoff $h$ which is a function defined on all possible values of the risky financial asset $S$ at maturity. If the market is complete, the payoff can be achieved by the investor. The market can be complete, for example as in previous section if it is dynamically complete in continuous time. It can also be complete in the context of a one period model, when European options of all strike prices are available on the market. The market may also be incomplete. In this case, the solution given in this section is only theoretical but is fruitful since this theoretical optimal payoff can be approximated by combinations of financial assets truly available on the market.

\subsubsection{The uninsured portfolio}

This section provides generalizations of results established by Brennan and Solanki (1981) or Carr and Madan (2001), as exposed in Prigent (2006). Consider an investor who desires to maximize the expected utility of his portfolio terminal value. Under the standard condition of no-arbitrage, the assets prices are calculated under risk neutral probabilities. If markets exist for out-of-themoney European puts and calls of all strikes, then it implies the existence of an unique risk-neutral probability that may be identified from option prices (see Breeden and Litzenberger,1978). Otherwise, if there is no continuous time trading, generally the market is incomplete and one particular risk-neutral probability $\mathbb{Q}$ is used to price the options. It is also possible that stock prices change continuously but the market may be still dynamically incomplete. Again, it is assumed that one risk-neutral probability is selected. Assume that prices are determined under such measure $\mathbb{Q}$. Denote by $\frac{d \mathbb{Q}}{d \mathbb{P}}$ the Radon-Nikodym derivative 
of $\mathbb{Q}$ with respect to the historical probability $\mathbb{P}$. Denote by $N_{T}$ the discount factor and by $M_{T}$ the product $N_{T} \frac{d \mathbb{Q}}{d \mathbb{P}}$.

Due to the no-arbitrage condition, the budget constraint corresponds to the following relation:

$$
V_{0}=\mathbb{E}_{\mathbb{Q}}\left[h\left(S_{T}\right) N_{T}\right]=\mathbb{E}_{\mathbb{P}}\left[h\left(S_{T}\right) M_{T}\right]
$$

The investor has to solve the following optimization problem:

$$
\operatorname{Max}_{h} \mathbb{E}_{\mathbb{P}}\left[U\left(h\left(S_{T}\right)\right] \text { under } V_{0}=\mathbb{E}_{\mathbb{P}}\left[h\left(S_{T}\right) M_{T}\right]\right.
$$

To simplify the presentation, we assume that the function $h$ satisfies the following condition:

$$
\int_{\mathbb{R}^{+}} h^{2}(s) \mathbb{P}_{S_{T}}(d s)<\infty .
$$

This means that $h \in \mathbb{L}^{2}\left(\mathbb{R}^{+}, \mathbb{P}_{S_{T}}(d s)\right)$ where $\mathbb{L}^{2}\left(\mathbb{R}^{+}, \mathbb{P}_{S_{T}}(d s)\right)$ denotes the set of all measurable functions with square that are integrable on $\mathbb{R}^{+}$with respect to the distribution $\mathbb{P}_{S_{T}}(d s)$.

For each utility function $U$ is associated a functional $\Phi_{U}$ which is defined on the space $\mathbb{L}^{2}\left(\mathbb{R}^{+}, \mathbb{P}_{S_{T}}(d s)\right)$ by:

$$
\forall Y \in \mathbb{L}^{2}\left(\mathbb{R}^{+}, \mathbb{P}_{S_{T}}(d s)\right), \Phi_{U}(Z)=\mathbb{E}_{\mathbb{P}_{S_{T}}}[U(Y)]
$$

Function $\Phi_{U}$ is usually called the functional of Nemitski associated to $U$ (see for example Ekeland and Turnbull (1983) for its definition and basic properties).

Introduce the conditional expectation of $M_{T}$ with respect to the $\sigma$-algebra generated by $S_{T}$ and denote by $k$ the function such that $M_{T}=k\left(X_{T}\right)$. Suppose that $k \in \mathbb{L}^{2}\left(\mathbb{R}^{+}, \mathbb{P}_{S_{T}}\right)$.

We deduce the following result: ${ }^{5}$

Proposition 8 The optimization problem is reduced to:

$$
\begin{gathered}
\operatorname{Max}_{h \in \mathbb{L}^{2}\left(\mathbb{R}^{+}, \mathbb{P}_{S_{T}}\right)} \int_{\mathbb{R}^{+3}} U(h(s)) \mathbb{P}_{S_{T}}(d s) \\
\text { under } V_{0}=\int_{\mathbb{R}^{+}} h(s) k(s) \mathbb{P}_{S_{T}}(d s) .
\end{gathered}
$$

We deduce that the optimal payment $h^{e}$ is given by:

$$
h^{*}=J(\rho k),
$$

where $\rho$ is the Lagrange parameter such that $V_{0}=\int_{\mathbb{R}^{+}} J[\rho k(s)] k(s) \mathbb{P}_{S_{T}}(d s)$.

Assume for example that the cash and the obligation are non-stochastic. Then the properties of the optimal payment $h^{*}$ as a function of the benchmark

\footnotetext{
${ }^{5}$ See Prigent (2006).
} 
$S$ can be analyzed. Specifically, by assuming that $k$ is differentiable, from optimality conditions we deduce that the derivative of the optimal payoff is given by:

$$
h^{\prime}(s)=\left(-\frac{U^{\prime}(h(s))}{U^{\prime \prime}(h(s))}\right) \times\left(-\frac{k(s)^{\prime}}{k(s)}\right) .
$$

Since the utility function $U$ is concave, the marginal utility $U^{\prime}$ is decreasing. Thus its inverse function $J$ is also decreasing. This allows us to deduce:

Corollary 9 The optimal payoff $h^{*}$ is an increasing function of the benchmark $S_{T}$ if and only if the conditional expectation $k$ of $M_{T}$ under the $\sigma$-algebra generated by $S_{T}$ is a decreasing function of $S_{T}$. Note that, most of the time, $k$ is decreasing.

Introduce the risk tolerance $T_{o}(h(s))$ which is equal to the inverse of absolute risk aversion:

$$
T_{o}(h(s))=-\frac{U^{\prime}(h(s))}{U^{\prime \prime}(h(s))} .
$$

Clearly, $h^{\prime}(s)$ depends on risk tolerance. The profile of the optimal payoff can further examined. For this purpose, introduce the term $Y(s)=-\frac{k^{\prime}(s)}{k(s)}$.

We obtain:

Corollary 10 Suppose that $k$ is twice differentiable. Then, we have:

$$
h^{\prime \prime}(s)=\left[X^{\prime}(h(s))+\frac{Y^{\prime}(s)}{Y(s)^{2}}\right] \times\left[X(h(s)) Y^{2}(s)\right] .
$$

We deduce that the higher the risk tolerance, the higher the value $h "(s)$.

\subsection{The insured portfolio}

Now suppose that the investor wants a specific guarantee that can be due for instance to specific institutional constraints or due to an additional protection against risk. For example, if the interest rate is stochastic, such guarantee can be modeled by a function $h_{0}$ defined on all possible values of the benchmark $S_{T}$ : whatever the value of $S_{T}$, the investor wants to obtain a final value of the portfolio that is always above the floor $h_{0}\left(S_{T}\right)$. For example, if $h_{0}$ linear with $h_{0}(s)=a s+b$, then, when the value of the benchmark decreases, the investor is sure to find at least the capital $b$ (equal to a predetermined percentage of his initial investment), and, if the benchmark increases, the investor benefits from this growth at a rate $a$.

The optimal payoff with insurance constraints on the terminal value, is solution of the following problem:

$$
\begin{aligned}
& \operatorname{Max}_{h} \mathbb{E}_{\mathbb{P}}\left[U\left(h\left(S_{T}\right)\right]\right. \\
V_{0}= & \mathbb{E}_{\mathbb{P}}\left[h\left(S_{T}\right) M_{T}\right] \\
h\left(S_{T}\right) \geq & F_{T}=h_{0}\left(S_{T}\right)
\end{aligned}
$$


Note that the value of the initial investment $V_{0}$ must be higher than $\mathbb{E}_{\mathbb{P}}\left[h_{0}\left(S_{T}\right) M_{T}\right]$, when we impose the guarantee constraint.

To solve the optimization problem, we observe that, for a given initial investment $V_{0}^{*}$, the investor seeks to determine the portfolio $w$ solution of the following optimization problem:

$$
\operatorname{Max}_{w} \mathbb{E}_{\mathbb{P}}\left[U\left(V_{T}\right)\right] \text { under } V_{T} \geq F_{T} .
$$

Due to the completeness of the market, this problem is equivalent to the following problem (see Cox-Huang, 1989):

$$
\begin{gathered}
\operatorname{Max}_{V_{T}} \mathbb{E}_{\mathbb{P}}\left[U\left(V_{T}\right)\right] \\
\text { under } V_{T} \geq F_{T} \text { and } V_{0}^{*}=\mathbb{E}_{\mathbb{P}}\left[V_{T} M_{T}\right] \geq \mathbb{E}_{\mathbb{P}}\left[F_{T} M_{T}\right] .
\end{gathered}
$$

The optimal solution $V_{T}^{*}$ of problem (25) is given by the maximum between the floor $F_{T}$ and the solution $V_{T}^{e}$ of the problem without constraint for an initial investment $V_{0}^{e}$ such as $V_{0}^{*}=\mathbb{E}_{\mathbb{P}}\left[\operatorname{Max}\left(V_{T}^{e}, F_{T}\right) M_{T}\right]$. Equivalently, this solution can be interpreted as a combination of the portfolio $V_{T}^{e}$ and a put written on it with strike price equal to the floor or as a combination of the floor and the portfolio $V_{T}^{e}$ :

$$
V_{T}^{*}=V_{T}^{e}+\left(F_{T}-V_{T}^{e}\right)^{+}=F_{T}+\left(V_{T}^{e}-F_{T}\right)^{+} .
$$

\subsection{The Brownian motion case}

In what follows, we recall the main results in the Brownian motion case. We note in particular that both dynamic and static approaches lead to the same optimal value of the portfolio, the difference being only in how to cover the optimal portfolio (dynamic versus static hedging).

Let $k$ be the density of $\frac{d \mathbb{Q}}{d \mathbb{P}}$ with respect to the $\sigma$-algebra generated by $W_{T}$.

Notations:

$$
\begin{aligned}
\theta & =\frac{\mu-r}{\sigma} \text { (Sharpe ratio), } \\
A & =-\frac{1}{2} \theta^{2} T+\frac{\theta}{\sigma}\left(\mu-\frac{1}{2} \sigma^{2}\right) T, \\
\chi & =e^{A}\left(S_{0}\right)^{\frac{\theta}{\sigma}}, \kappa=\frac{\theta}{\sigma} .
\end{aligned}
$$

Lemma 11 The density $k$ of the risk neutral probability is given by:

$$
\eta_{T}=k\left(S_{T}\right) \text { with } k(s)=\chi s^{-\kappa} .
$$

Then, we can express the optimal portfolio as a function of benchmark $S_{T}$.

Proposition 12 The optimal solution in the CRRA case with $U(x)=\frac{x^{1-\gamma}}{1-\gamma}$ $(\gamma \neq 1)$ is given by:

$$
V_{T}^{*}=h^{*}\left(S_{T}\right)=c \cdot S_{T}^{\frac{\kappa}{\gamma}}
$$


where the power $\frac{\kappa}{\gamma}$ of $S_{T}$ is equal to the Sharpe type ratio $\kappa=\frac{\mu-r}{\sigma^{2}}$ multiplied by the inverse of the relative risk aversion. The higher the Sharpe type ratio (the higher the market performance) or the lower the relative risk aversion (risk attitude), the more convex the optimal payoff function (the higher the risk-taking).

According to the financial parameter values $\mu$ and $\sigma$, the power of $S_{T}$ is or not higher than 1, which leads or not to the convexity of the payoff function.

The concavity / convexity of the optimal payoff is determined by comparing:

- The relative risk aversion $\gamma$.

- The ratio $\kappa=\frac{\mu-r}{\sigma^{2}}$, which is the Sharpe ratio, divided by the volatility $\sigma$.

This gives us the following characterization:

i) $h^{*}(s)$ is concave if $\kappa<\gamma$.

ii) $h^{*}(s)$ is linear if $\kappa=\gamma$.

iii) $h^{*}(s)$ is convex if $\kappa>\gamma$.

This result is illustrated in the following figure, where we modify the expected instantaneous rate of return $\mu$ of the risky asset which directly influences the Sharpe type ratio $\kappa$, the relative risk aversion being fixed in this example at the level $\gamma=0.9$.

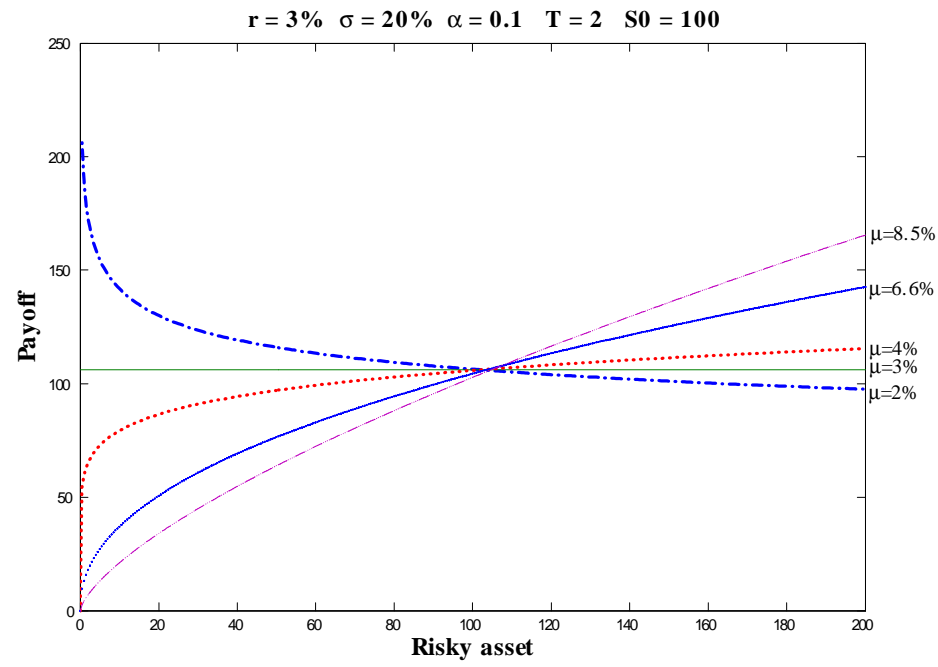

Figure 1: Optimal payoff for various values of $\mu$ 
We note that:

- The payoff $h^{*}(s)$ is concave if $\kappa<\gamma$, for example, for $\mu=4 \%$ on previous figure. When the market is bearish, the investor wishes to recover a significant amount, thereby forfeits some gains in a potential bullish market.

- The payoff $h^{*}(s)$ is linear if $\kappa=\gamma$ (here, this case corresponds to $\mu=$ $6.6 \%)$. The investor invests in that case only on the risky asset which is the underlying $S$ itself. He does not use options.

- The payoff $h^{*}(s)$ is convex if $\kappa>\gamma$ (for example, $\mu=8.5 \%$ ). Symmetrically to the first case, the investor is more exposed to market risk, to take full advantage of a possible increase, but he is less protected against a market decline.

\subsubsection{The insured portfolio}

For this particular example, the guarantee can be modeled as a function $h_{0}$ defined on the risky asset value $S_{T}$. Whatever the values of $S_{T}$, the investor requires that the value of his portfolio is above the value $h_{0}\left(S_{T}\right)$. For example, the guarantee $h_{0}$ can be linear in $S_{T}$ such as $h_{0}(s)=a s+b$. The guaranteed amount is equal to $b$ and, if the benchmark increases, he can benefit from this rise through the proportion $a$.

The value of the optimal solution is the solution of the following optimization problem:

$$
\begin{aligned}
& \operatorname{Max}_{h} \mathbb{E}_{\mathbb{P}}\left[U\left(h\left(S_{T}\right)\right]\right. \\
V_{0}= & \mathbb{E}_{\mathbb{P}}\left[h\left(S_{T}\right) g\left(S_{T}\right)\right] \\
h\left(S_{T}\right) \geq & h_{0}\left(S_{T}\right)
\end{aligned}
$$

Note also that the value of the initial investment $V_{0}$ must be greater than $\mathbb{E}_{\mathbb{P}}\left[h_{0}\left(S_{T}\right) M_{T}\right]$, when we impose the constraint of guarantee. As mentioned in previous section, the first-order optimality condition leads to the following result:

Proposition 13 The optimal payoff in the presence of the guarantee constraint $h_{0}$, is given by:

$$
h^{* *}\left(S_{T}\right)=\operatorname{Max}\left[h_{0}\left(S_{T}\right), h^{*}\left(S_{T}\right)\right] .
$$

In the case of CRRA utility and of a linear guarantee constraint, we obtain the following optimal solution:

$$
h^{* *}\left(S_{T}\right)=h_{0}\left(S_{T}\right)+\operatorname{Max}\left[\widetilde{c} . S_{T}^{\frac{\kappa}{\gamma}}-h_{0}\left(S_{T}\right), 0\right] .
$$


Remark 14 As it can be seen, the graph of the function of optimal payment under linear guarantee constraint $\left(h_{0}(s)=a s+b\right)$, changes from concavity to convexity according in particular to the relative risk aversion.

The optimal solution $h^{* *}$ is similar to that of the case without constraint, except when $h^{* *}$ is equal to the constraint $h_{0} . h^{* *}(s)$ is an increasing function of $s$. Its form depends only on the comparison between $\gamma$, for each investor, and $\kappa$, parameter that can be seen as a Sharpe ratio per unit of volatility, thus synthesizing alone market parameters $\mu, r$ and $\sigma$.

In the following figures, the guarantee corresponds only to a fixed proportion $90 \%\left(a=0 ; b=0.9 \times V_{0}\right)$. According to the order of magnitude of the volatility $\sigma$, we obtain a concave (relatively high $\sigma$ ) or convex (relatively low $\sigma$ ) profile.

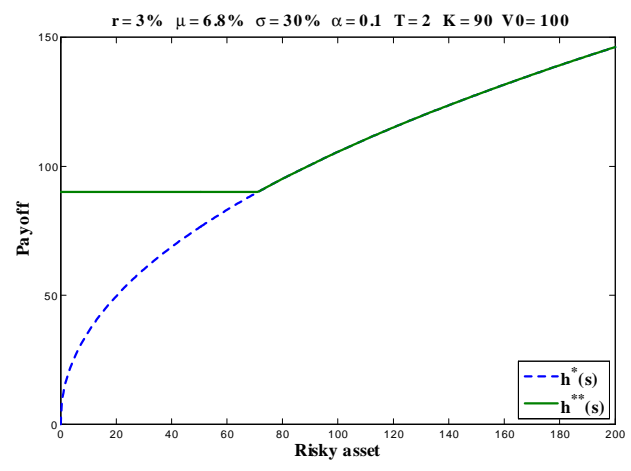

(a) Optimal payoff:concave case

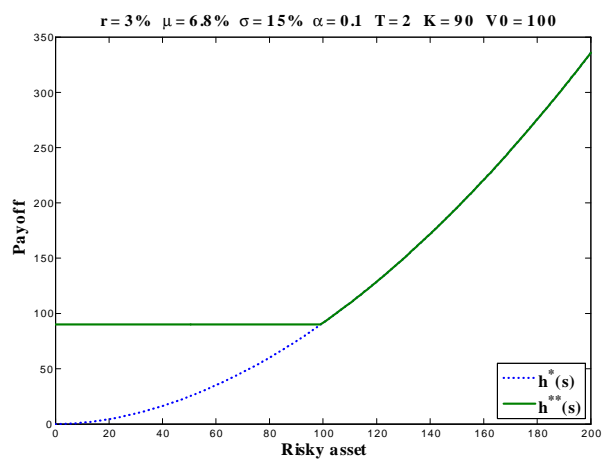

(b) Optimal payoff: convex case

Figure 2: Optimal payoff

The previous results allow as to completely determine the optimal portfolio value expression. 


\subsection{Optimal portfolio value within Johnson distributions}

The investor maximizes his expected utility:

$$
\left.\operatorname{Max}_{h} \mathbb{E}_{[}\left[U\left[V_{T}\right)\right]\right],
$$

where $V_{T}$ designates the portfolio payoff at maturity $T$.

\subsubsection{The risk-neutral probability within Johnson distributions}

We adopt the same approach as Buhlman et al. (1996) to determine the riskneutral density probability from the Girsanov's theorem. From Harrison and Kreps (1979) and Harrison and Pliska (1981), we deduce that the option prices are equal to the discounted expectations of their payoff functions under the (unique) risk-neutral probability denoted by $\mathbb{Q}$. The measure $\mathbb{Q}$ is equivalent to $\mathbb{P}$ and such that the discounted price $\tilde{S}$ (with $\tilde{S}_{t}=e^{-r t} S_{t}$ ) is a martingale under $\mathbb{Q}$. To determine this measure, we must calculate the Radon-Nikodym process $\eta$ defined by $\eta_{t}=\mathbb{E}\left[\frac{d \mathbb{Q}}{d \mathbb{P}} \mid \mathcal{F}_{t}\right]$ which is strictly positive. Introduce the process $\tau$ such as $\tau_{t}=\int_{0}^{t} \frac{d \eta_{s}}{\eta_{s-}}$ where $\eta_{t}=\eta_{0} \mathcal{E}\left[\tau_{t}\right]$. As the process $\tau$ is a local martingale with respect to the filtration generated by the Brownian motion $W$, it can be written as:

$$
\tau_{t}=\int_{0}^{t} \beta_{s} d W_{s}
$$

where the process $\beta$ fulfills an integrability condition (see Jacod-Shiryaev, 2003 Ch III, 4.24). The process $\beta$ determines entirely the risk-neutral probability $\mathbb{Q}$.

Proposition 15 The risk-neutral probability is characterized by its Radon-Nikodym derivative $\eta$, given by:

$$
\eta_{t}=\mathcal{E}\left[\int_{0}^{t} \beta_{s} d W_{s}\right]
$$

with $\mathbb{E}\left[\eta_{T}=1\right]$ and $\beta$ satisfies the following Girsanov's equation:

$$
r t=A_{t}^{R}+\int_{0}^{t} \beta_{s} b\left(s, X_{s}\right) d s
$$

This is equivalent to:

$$
r t=A_{t}^{X}+1 / 2 \int_{0}^{t} b^{2}\left(s, X_{s}\right) d s+\int_{0}^{t} \beta_{s} b\left(s, X_{s}\right) d s .
$$

We deduce that:

$$
\beta_{t}=-\frac{-r+d A_{t}^{X} / d t+1 / 2 b^{2}\left(t, X_{t}\right)}{b\left(t, X_{t}\right)} .
$$

Proof. See Buhlman et al. (1996) who use crucially the fact that the process $\left(\eta_{t} \tilde{S}_{t}\right)_{t}$ is a $\mathbb{P}$-martingale, i.e. its bounded variation part is equal to zero. 
Denote by $\widetilde{R}$ the discounted value of the process $R$ defined in Relation (9). Recall also the Yor's formula for two semimartingales $\widetilde{R}$ and $\tau$ :

$$
\mathcal{E}\left[\widetilde{R}_{t}\right] \mathcal{E}\left[\tau_{t}\right]=\mathcal{E}\left[\widetilde{R}_{t}+\tau_{t}+[\widetilde{R}, \tau]_{t}\right]
$$

where $[\widetilde{R}, \tau]$ denotes the quadratic variation of the two processes $\widetilde{R}_{t}$ and $\tau$.

Consequently, the process $\left(\eta_{t} \tilde{S}_{t}\right)_{t}$ is a $\mathbb{P}$-martingale if and only if the bounded variation component of process $\widetilde{R}_{t}+\tau_{t}+[\widetilde{R}, \tau]_{t}$ is equal to 0 .

Therefore we get the following condition:

$$
A_{t}^{R}-r t+\left[\int_{0}^{t} b\left(s, X_{s}\right) d W_{s}, \int_{0}^{t} \beta_{s} d W_{s}\right]=0 .
$$

By using the property $\left[\int \theta_{s} d W_{s}, \int \vartheta_{s} d W_{s}\right]_{t}=\int_{0}^{t} \theta_{s} \vartheta_{s} d s$, we deduce the Girsanov's equation:

$$
A_{t}^{R}-r t+\int_{0}^{t} b\left(s, X_{s}\right) \beta_{s} d s=0,
$$

from which, we deduce previous relations (36) and (??), hence the expression (38) of the risk premium $\beta$.

Remark 16 We find the standard case when the process $\left(X_{t}-\left[\mu-1 / 2 \sigma^{2}\right] t\right) / \sigma$ is the Brownian motion (Gaussian standard distribution in place of the Johnson's probability law):

$$
\beta_{t}=-\frac{\mu-r}{\sigma} .
$$

Given that the no-arbitrage price of any contingent asset $f\left(S_{T}\right)$ is equal at any time $t$ to $e^{-r t} \mathbb{E}\left[f\left(S_{T}\right) \mid \mathcal{F}_{t}\right]$, we need to know the probability of the risky asset price under the risk-neutral probability $\mathbb{Q}$ associated to process $\beta$. By using the calculation of local characteristics (see Jacod-Shiryaev,2003 chap III), we can establish that the new characteristics $[\tilde{B}(h), \tilde{C}]$ of $X$ under risk-neutral probability $\mathbb{Q}$ associated to process $\beta$ are given by:

$$
\left\{\begin{array}{l}
\widetilde{A}_{t}^{X}=A_{t}^{X}+1 / 2 \int_{0}^{t} d\left\langle M^{X}, M^{X}\right\rangle_{s}, \\
\tilde{C}_{t}^{X}=\left\langle M^{X}, M^{X}\right\rangle_{t} .
\end{array}\right.
$$

Proposition 17 The first order condition allows the determination of optimal portfolio value:

$$
V_{T}^{*}=J\left[\rho \eta_{T}\right],
$$

where $J$ is the inverse of the marginal utility $U$ and $\rho$ is the Lagrange parameter associated to the budget constraint. 
Proof. The result is analogous to the standard case (see the relation 14 ). In fact, the function $g$ is supposed to be invertible. Information delivered by the observation of $S$ is also the same as that of Brownian motion. We can therefore use the dynamic completeness of the financial market.

Proposition 18 The optimal portfolio value $V_{T}^{*}$ is given by:

$$
V_{T}^{*}=J\left[\rho \eta_{T}\right],
$$

where $\eta_{t}$ is defined by:

$$
\eta_{t}=\eta_{0} \mathcal{E}\left[\tau_{t}\right]=\eta_{0} \exp \left[-\frac{1}{2} \int_{0}^{t} \beta_{s}^{2} d s+\int_{0}^{t} \beta_{s} d W_{s}\right]
$$

with process $\beta$ defined in (38).

Corollary 19 The optimal solution depends on the whole trajectory of the risky asset $S .{ }^{6}$

Corollary 20 (Case of constant coefficients) When the parameters $\gamma_{t}, \delta_{t}, \xi_{t}$ and $\lambda_{t}$ are constant, we obtain a simplified version of the optimal portfolio as function of the risky asset:

$$
V_{T}^{*}=J\left[\rho \eta_{T}\right]
$$

where $\eta_{T}$ is defined by:

$$
\eta_{T}=\eta_{0} \mathcal{E}\left[\tau_{T}\right]=\eta_{0} \exp \left[-\frac{1}{2} \int_{0}^{T} \beta_{s}^{2} d s+\int_{0}^{T} \beta_{s} d W_{s}\right],
$$

with:

$$
\beta_{t}=
$$

$$
\frac{\delta}{\lambda} r g^{\prime}\left(\frac{\ln \left(\frac{S_{t}}{S_{0}}\right)-\xi}{\lambda}\right)+\frac{1}{2 \delta}\left(\frac{g^{\prime \prime}}{g^{\prime 2}}\right)\left(\frac{\ln \left(\frac{S_{t}}{S_{0}}\right)-\xi}{\lambda}\right)-1 / 2 \frac{\lambda}{\delta}\left[1 / g^{\prime}\left(\frac{\ln \left(\frac{S_{t}}{S_{0}}\right)-\xi}{\lambda}\right)\right]
$$

and:

$$
\begin{gathered}
d W_{t}= \\
\frac{\delta}{\lambda} g^{\prime}\left(\frac{\ln \left(\frac{S_{t}}{S_{0}}\right)-\xi}{\lambda}\right)\left(\frac{d S_{t}}{S_{t}}\right)+\frac{1}{2 \delta} \frac{g^{\prime \prime}\left(\frac{\ln \left(\frac{S_{t}}{S_{0}}\right)-\xi}{\lambda}\right)}{\left[g^{\prime}\left(\frac{\ln \left(\frac{S_{t}}{S_{0}}\right)-\xi_{t}}{\lambda_{t}}\right)\right]^{2}}
\end{gathered}
$$

\footnotetext{
${ }^{6}$ See Proof in Appendix B.
} 
Corollary 21 Suppose that the utility function is of CRRA type:

$$
U(x)=\frac{x^{1-\gamma}}{1-\gamma} .
$$

In this case, we obtain:

$$
V_{T}^{*}=\rho^{-\frac{1}{\gamma}} \exp \left[\frac{1}{2 \gamma} \int_{0}^{t} \beta_{s}^{2} d s-\frac{1}{\gamma} \int_{0}^{t} \beta_{s} d W_{s}\right] .
$$

For the geometric Brownian motion case, we get: (see Formula 29)

$$
V_{T}^{*}=c S_{T}^{\frac{-\beta_{b}}{\sigma} \frac{1}{\gamma}}
$$

with $\beta_{b}$ corresponding to the standard Brownian case.

\subsubsection{The portfolio insurance case}

In this particular context of Johnson law, we examine the optimal solution in the presence of a guarantee constraint. Such a guarantee can be modeled as a function $h_{0}$ defined now both on the value of the risky asset $S_{T}$. Whatever the values of $S_{T}$, the investor requires that the value of his portfolio will always be above the value $h_{0}\left(S_{T}\right)$. The solution is deduced from previous section, since its general form does not depend on the type of law (i.e. on the choice of function $g)$.

Proposition 22 The optimal payoff, in presence of constraint guarantee $h_{0}$, is given by:

$$
h^{* *}\left(S_{T}\right)=\operatorname{Max}\left[h_{0}\left(S_{T}\right), h^{*}\left(S_{T}\right)\right]=h_{0}\left(S_{T}\right)+\operatorname{Max}\left[h^{*}\left(S_{T}\right)-h_{0}\left(S_{T}\right), 0\right] .
$$

In the CRRA utility case and with a linear guarantee constraint, we obtain the following optimal solution: $h^{* *}\left(S_{T}\right)=$

$$
h_{0}\left(S_{T}\right)+\operatorname{Max}\left[\tilde{\rho}^{-\frac{1}{\gamma}} \exp \left[+\frac{1}{2 \gamma} \int_{0}^{T} \beta_{s}^{2} d s-\frac{1}{\gamma} \int_{0}^{T} \beta_{s} d W_{s}\right]-h_{0}\left(S_{T}\right), 0\right],
$$

where $\widetilde{\rho}$ is the Lagange coefficient corresponding to the unscontrained solution but with an initial invested amount $\widetilde{V}_{0}$ smaller than $V_{0}$.

For the geometric Brownian motion case, we get:

$$
\begin{gathered}
h^{* *}\left(S_{T}\right)= \\
h_{0}\left(S_{T}\right)+\operatorname{Max}\left[\widetilde{\rho}^{-\frac{1}{\gamma}} \widetilde{c}_{T} S_{T}^{\frac{\beta_{b}}{\sigma} \frac{1}{\gamma}}-h_{0}\left(S_{T}\right), 0\right] .
\end{gathered}
$$

In contrast to the case of Brownian motion, we note that the optimal portfolio depends on the whole trajectory of the risky asset and not only of its value at maturity $T$. Therefore, this portfolio requires a dynamic hedging ${ }^{7}$ while that of the Brownian case can be potentially obtained by means of an infinity of European options.

\footnotetext{
${ }^{7}$ This hedging strategy can be determined by Clark-Haussmann-Ocone formula (see Aase et al., 2000; Clark, 1970).
} 


\section{Numerical illustration}

\subsection{Johnson parameters estimation}

Different estimation methods are proposed. The most used methods are: the method of moments by using the algorithm of Hill et al. (1976), the method of Wheeler and Robert (1980) and the method of Slifker and Shapiro (1980).

\subsubsection{The method of moments}

The method of moments consists in estimating the parameters on the basis of the first four moments of the empirical distribution. It is a recursive procedure allowing the determination of the parameters $\gamma$ and $\delta$ in a first step, then the determination of the values of $\xi$ and $\lambda$ by a simple linear regression. In fact, for each family of distributions, Johnson (1949) expresses the four moments as function of the four parameters. But, before proceeding with parameter estimation, it is necessary to know the distribution family that best fits the data. This is achieved by first calculating the skewness and kurtosis of the data series (in our empirical study, the series corresponds to the hedge funds logarithmic returns), then by placing the point $p$ of coordinates (skewness, kurtosis) on a plan which has the abscissa axis as skewness and kurtosis as ordinate axis. We plot the curve $C$ of the log-normal function on the same plane and we observe the point location $p$ with respect to the curve $C$, distribution is of the type $S_{U}$, if it is located on the curve $C$, so this is a log-normal distribution and if it is below the curve $C$, the distribution is of the type $S_{B}$. Hill et al. (1976) have developed an algorithm that determines the type of function $g($.$) and estimate$ the parameters by the moments method. The moment method seems very useful since it allows to construct distributions from the first four moments. It thus allows to measure the impact of asymmetry and flattening on the investment decision. The criticism addressed to this method is the estimation bias of the moments of higher orders when the sample size is small. The figure (3) illustrates the choice of the Johnson distribution by the moments method.

\subsubsection{The quartiles method}

To solve the problems related to the moments method, Wheeler and Robert (1980) propose an extension of the quantiles method introduced by Aitchison and Brown (1957) to estimate the parameters of the lognormal distribution. The theoretical foundation of using the quantile approach is that each member of distribution is identified by the distance between the tails and the central part of the distribution. The authors suggest that using this approach for the determination of parameters is much better they are determined by their skewness and kurtosis. In their study, Wheeler and Robert (1980) give formulas that allow the parameters estimation.

Slifker and Shapiro (1980) also depart from the same principle and give an extension to the previous method. They consider that four rather than five 


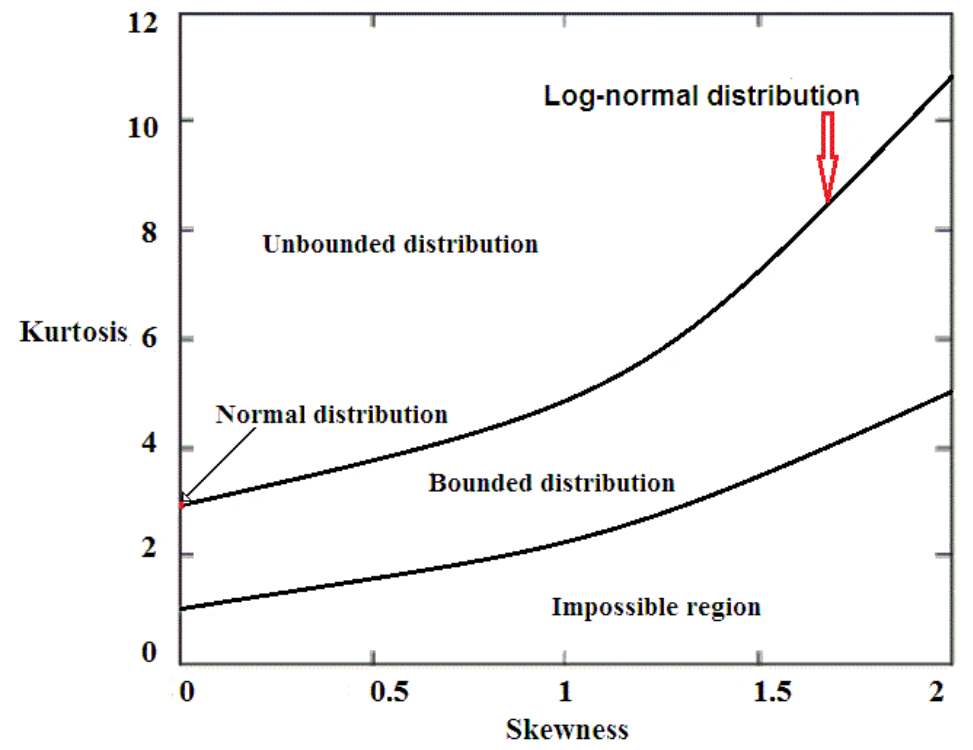

Figure 3: Determination of distribution type by the method of moments

quantiles are sufficient to estimate the four distribution parameters. They also propose a new method to choose the family distribution and the explicit form of the parameters for each distribution family. In what follows, we present the quartile method proposed by Slifker and Shapiro (1980). To apply this method we must proceed as follows:

1. Choose a value $z>0$ and deduce four points $\psi$ such as $\psi=-3 z,-z, z, 3 z$.

2. From the table of normal distribution, we determine the probabilities $p_{\psi}$ corresponding at points $\psi$.

3. From the data series, we estimate the quantiles $x^{(i)}$ corresponding at probabilities $p_{\psi}$ by using the relationship $\frac{i-0.5}{n}=p_{\psi}$ and define $x_{\psi}=x^{(i)}$. therefore $x_{\psi}$ is the $i^{t h}$ observation. The value of $i$ is obtained by interpolation. Here $n$ corresponds to the number of observations.

4. From obtained value, we determine the values of $m, n$ and $p$ such as $m=$ $x_{3 z}-x_{z}, n=x_{-z}-x_{-3 z}$ and $p=x_{z}-x_{-z}$.

5. The penultimate step is determine the type of distribution on the basis the following choice criterion:

$$
m n p^{-2}>1 \quad S_{U}
$$




$$
\begin{array}{ll}
m n p^{-2}<1 & S_{B} \\
m n p^{-2}=1 & S_{L}
\end{array}
$$

Once the type of distribution is determined, we estimate the parameters using the formulas given by Slifker and Shapiro (1980).

Criticism of the quantiles method estimation is essentially the fact that the results depend on the choice of $z$. Slifker and Shapiro (1980) propose that the value of $z$ is around 0.5 . They choose a value equal to 0.524 .

\subsection{Estimation of hedge funds returns with Johnson sys- tem}

Our study is conducted from the indexes Credit Suisse / Tremont Hedge Fund (CSFB / Tremont) provided by Credit Suisse. These indexes present the monthly returns of the ten main families of alternative strategies identified by this data provider. These indexes are Convertible Arbitrage (CA), Dedicated Short Bias (DSB), Emerging Markets (EM), Equity Market Neutral (EMN), Event Driven (ED), Fixed Income Arbitrage (FIA), Global Macro (GM), Long / Short Equity (LSE), Managed Futures (MF) and Multi-Strategy (MF). The global index constructed from the monostrategy indices, is used to represent all hedge fund strategies. These indexes are constructed using the TASS database which includes more than 3000 U.S. and offshore hedge funds. The characteristic of these indexes is that they are known by the transparency guaranteed by Credit Swiss in their communication and construction mode.

These indexes include only funds having at least $\$ 10$ million under management and providing audited financial reports. After this filtering, 400 funds on average are retained. The procedure for selecting funds. is renewed quarterly to update calculations. In fact, if a fund no longer satisfies expectations of financial reporting, it is systematically excluded. As to the classification of funds by major styles of strategy, it is realized on the basis of declarations of the managers. Funds are then weighted by their capitalization and presented net of commissions.

The study of indexes is conducted on the period from April 1994 until December 2013, which gives us the possibility to work on 240 monthly observations. This period covers a number of financial crises certainly including the current crisis.

We estimate the family and the parameters of Johnson distributions for the 10 sub-indexes of hedge funds by the three methods. Since we are in presence of three different estimation methods, we make a fit test $\chi^{2}$ to differentiate them. 
Table 1: Distribution type and Johnson parameters estimated by the moments method

\begin{tabular}{lrrrcc}
\hline & \multicolumn{1}{c}{$\gamma$} & \multicolumn{1}{c}{$\xi$} & \multicolumn{1}{c}{$\lambda$} & \multicolumn{1}{c}{ Type } \\
\hline Convertible Arbitrage & 0.8394 & 1.2845 & 0.0178 & 0.0231 & $\mathrm{SU}$ \\
Dedicated Short Bias & -1.5101 & 2.5148 & -0.0674 & 0.0925 & $\mathrm{SU}$ \\
Event Driven & 1.2321 & 1.4236 & 0.0288 & 0.0168 & $\mathrm{SU}$ \\
Emerging Markets & 0.4364 & 1.4486 & 0.0283 & 0.0554 & $\mathrm{SU}$ \\
Equity Market Neutral & 0.4745 & 1.4760 & 0.0322 & 0.0617 & $\mathrm{SU}$ \\
Fixed Income Arbitrage & 1.8461 & 1.3189 & 0.0259 & 0.0084 & $\mathrm{SU}$ \\
Global Macro & -0.0221 & 1.4753 & 0.0087 & 0.0312 & $\mathrm{SU}$ \\
Long/Short Equity & -0.0259 & 1.6319 & 0.0071 & 0.0443 & $\mathrm{SU}$ \\
Managed Futures & 0 & 1 & 0.0333 & 0.0047 & $\mathrm{SN}$ \\
Multi-Strategy & 0.9981 & 1.7174 & 0.0235 & 0.0231 & $\mathrm{SU}$ \\
\hline
\end{tabular}

Table 2: Distribution type and Johnson parameters estimated by the method of Wheeler and Robert (1980)

\begin{tabular}{lrrrcc}
\hline & \multicolumn{1}{c}{$\gamma$} & \multicolumn{1}{c}{$\xi$} & \multicolumn{1}{c}{$\lambda$} & Type \\
\hline Convertible Arbitrage & 0.2850 & 0.9563 & 0.0128 & 0.0116 & $\mathrm{SU}$ \\
Dedicated Short Bias & 0 & 4.8091 & -0.2286 & 0.2197 & $\mathrm{SL}$ \\
Event Driven & 0.6080 & 1.3611 & 0.0202 & 0.0201 & $\mathrm{SU}$ \\
Emerging Markets & 0.4059 & 1.1549 & 0.0273 & 0.0382 & $\mathrm{SU}$ \\
Equity Market Neutral & 0.3987 & 1.1663 & 0.0291 & 0.0439 & $\mathrm{SU}$ \\
Fixed Income Arbitrage & 0.3502 & 1.1513 & 0.0099 & 0.0100 & $\mathrm{SU}$ \\
Global Macro & -0.0087 & 1.1211 & 0.0093 & 0.0188 & $\mathrm{SU}$ \\
Long/Short Equity & -0.0908 & 1.5549 & 0.0052 & 0.0402 & $\mathrm{SU}$ \\
Managed Futures & 0 & 1 & 0.0041 & 0.0325 & $\mathrm{SN}$ \\
Multi-Strategy & 0.3350 & 1.0656 & 0.0133 & 0.0125 & $\mathrm{SU}$ \\
\hline
\end{tabular}

Tables 1,2 and 3 show that most of hedge fund returns have a $S_{U}$ Johnson distribution. We also note that the distribution type and parameter values vary depending on the estimation method used. In fact, for the index Dedicated Short Bias, by the method of Wheeler and Robert (1980) it is estimated that distribution is bounded whereas with the method of moments and that of Slifker and Shapiro (1980), it is estimated that distribution is not bounded. In order to classify these three methods, we used the fit test $\chi^{2}$. With this test, the best method is that which minimizes the value of $\chi^{2}$.

Table (4) shows that for each index, corresponds a particular method of estimation. 
Table 3: Distribution type and Johnson parameters estimated by the method of Slifker and Shapiro (1980)

\begin{tabular}{lrcrcr}
\hline & $\gamma$ & $\delta$ & $\xi$ & \multicolumn{1}{c}{$\lambda$} & Type \\
\hline Convertible Arbitrage & 0.2558 & 0.9207 & 0.0125 & 0.0116 & $\mathrm{SU}$ \\
Dedicated Short Bias & -1.9022 & 1.4407 & -0.1321 & 0.0683 & $\mathrm{SL}$ \\
Event Driven & 0.7837 & 1.5433 & 0.0220 & 0.0219 & $\mathrm{SU}$ \\
Emerging Markets & 0.2825 & 1.3488 & 0.0213 & 0.0468 & $\mathrm{SU}$ \\
Equity Market Neutral & 0.2574 & 1.3361 & 0.0210 & 0.0496 & $\mathrm{SU}$ \\
Fixed Income Arbitrage & 0.2444 & 0.9018 & 0.0090 & 0.0074 & $\mathrm{SU}$ \\
Global Macro & -0.0045 & 0.9383 & 0.0096 & 0.0140 & $\mathrm{SU}$ \\
Long/Short Equity & -0.0434 & 1.5932 & 0.0070 & 0.0407 & $\mathrm{SU}$ \\
Managed Futures & -0.0141 & 2.4468 & 0.0045 & 0.1308 & $\mathrm{SU}$ \\
Multi-Strategy & 0.3745 & 1.2326 & 0.0139 & 0.0157 & $\mathrm{SU}$ \\
\hline
\end{tabular}

Table 4: Values of $\chi^{2}$ test according different estimation methods

\begin{tabular}{lrrr}
\hline & Moments & Wheeler \& Robert & Slifker \& Shapiro \\
\hline Convertible Arbitrage & 15.7779 & 1.5259 & 1.2867 \\
Dedicated Short Bias & 7.1041 & 16.2136 & 15.9684 \\
Event Driven & 13.1077 & 6.3583 & 7.9988 \\
Emerging Markets & 6.3238 & 10.2190 & 11.7220 \\
Equity Market Neutral & 5.8309 & 10.0031 & 11.8772 \\
Fixed Income Arbitrage & 59.0119 & 2.3502 & 2.5957 \\
Global Macro & 16.0726 & 4.7018 & 2.1119 \\
Long/Short Equity & 4.0751 & 2.4113 & 2.5970 \\
Managed Futures & 3.3392 & 3.3392 & 3.0278 \\
Multi-Strategy & 6.8135 & 2.6522 & 0.5765 \\
\hline
\end{tabular}

\subsection{Non parametric estimations of financial assets returns}

As we have already seen, when the financial asset returns are not Gaussian, we can use the Johnson system to estimate their distribution laws. But this system of distributions can in no way cover all the laws of distributions. There is also another problem with the estimation by this system (as with any other parameter estimation) is the bias associated with parameter estimates. For this, we propose to estimate the distribution of not Gaussian financial assets returns with a nonparametric estimation. To conduct a nonparametric estimation, we consider that any random variable with distribution function invertible which we do not know the distribution law, can be transformed into a random variable that follows a standard normal distribution.

Lemma 23 Let $X$ be a random variable which we want to estimate the distribution law. Then we can define a function l such that:

$$
X=l(\varepsilon),
$$


and

$$
\varepsilon=l^{-1}(X)
$$

where $l$ is a strictly increasing function and $\varepsilon \rightsquigarrow N(0,1)$.

Proof. Let $F_{X}(x)$, the distribution function of $X$ :

$$
\begin{gathered}
F_{X}(x)=\mathbb{P}[X \leq x], \\
F_{X}(x)=\mathbb{P}[l(\varepsilon) \leq x] .
\end{gathered}
$$

Since $l$ is strictly increasing, we have:

$$
F_{X}(x)=\mathbb{P}\left[\varepsilon \leq l^{-1}(x)\right] .
$$

Since $\varepsilon \rightsquigarrow N(0,1)$, we obtain:

$$
F_{X}(x)=N\left(l^{-1}(x)\right) .
$$

If $N^{-1}(x)$ is the inverse cumulative distribution function of the standard normal distribution, then the function $l^{-1}(x)$ has the following form:

$$
l^{-1}(x)=N^{-1}\left[F_{X}(x)\right] .
$$

Hence:

$$
l(x)=\left(N^{-1}\left[F_{X}(x)\right]\right)^{-1}=\left(\left[F_{X}(x)\right]\right)^{-1} \circ N .
$$

where $(.)^{-1}$ designates the inverse of a function (.).

Note that the estimate of the distribution function can be done using the empirical distribution function (see the nonparametric method of kernel for density estimation).

The function $\widehat{l}(x)$ is estimated, we can make polynomial interpolation to determine its form as a function of $x$.

Thus, we see that with a transformation of the normal distribution we can express the financial asset return according to a standard normal variable under fairly general conditions.

In what follows, we illustrate the non parametric estimation of a generalized Johnson distribution.

1. Figure (4) shows that estimated functions $l$ are non decreasing. Thus we can apply them to the standard Gaussian distribution. The function $l$ can be approximated by a polynomial interpolation. For example, for the Multi Strategy Index, we can provide several polynomial approximations of distinct orders depending on the accuracy of the approximation of $l$.

$$
\begin{aligned}
l(x) & =c+\alpha_{1} x+\alpha_{2} x^{2}+\alpha_{3} x^{3} \\
& =0.0071+0.0237 x-0.0019 x^{2}+0.0001 x^{3}
\end{aligned}
$$



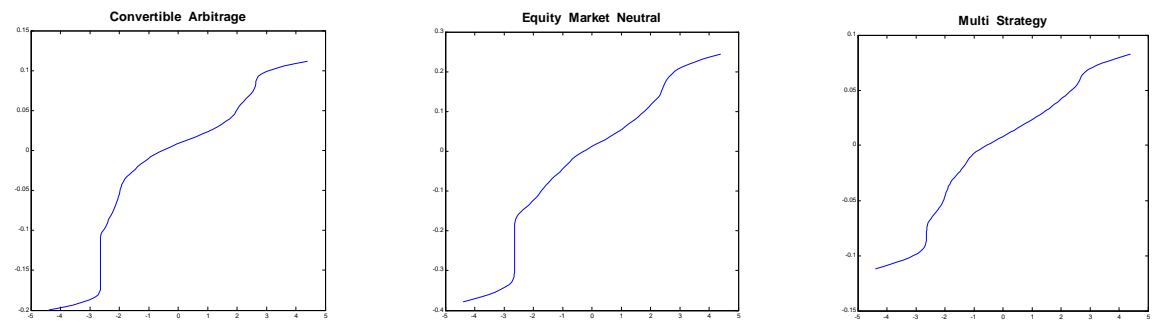

Figure 4: Non parametric estimations of functions 1

and

$$
\begin{aligned}
l(x) & =c+\alpha_{1} x+\alpha_{2} x^{2}+\alpha_{3} x^{3}+\alpha_{4} x^{4}+\alpha_{5} x^{5} \\
& =0.0113+0.0138 x-0.0041 x^{2}+0.0025 x^{3}+0.0001 x^{4}-0.0001 x^{5}
\end{aligned}
$$

Figures $(5,6,7)$ display the polynomial interpolations of function $l$ for degrees 3 and 5 and illustrate their comparison. Note that both are increasing as required to define generalized Johnson distributions for the three hedge fund types, namely the convertible arbitrage, equity market neutral and multi strategy indices. Using these approximations, we can simulate the values of different indices by means of simulation of the standard Gaussian distribution.
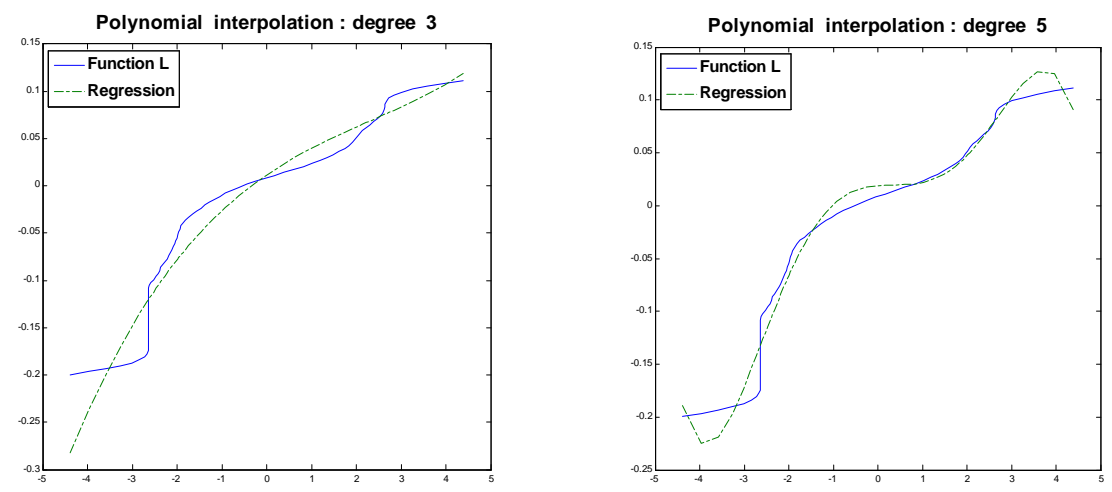

Figure 5: Polynomial interpolation of function 1 of the Convertible Arbitrage index 

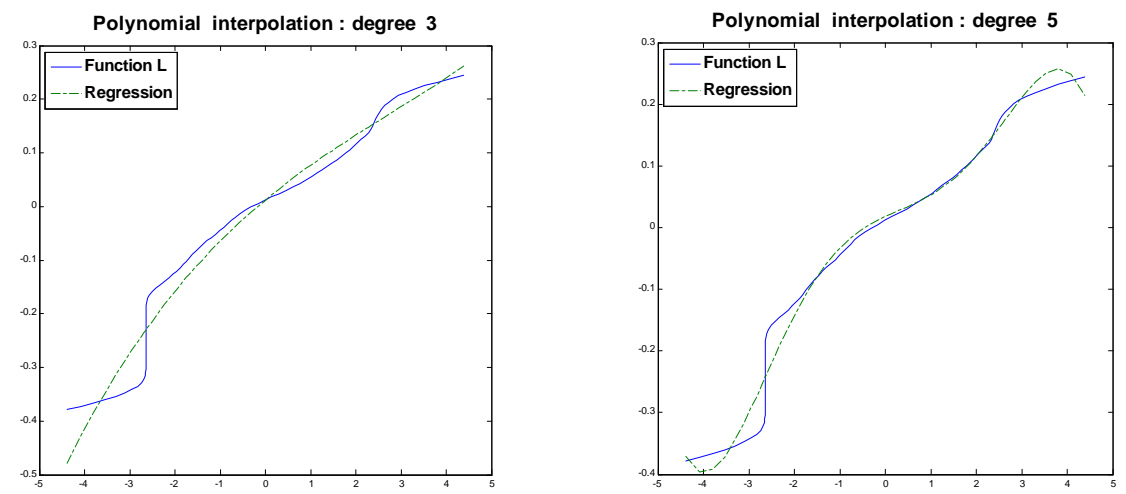

Figure 6: Polynomial interpolation of function 1 of the Equity Market Neutral index
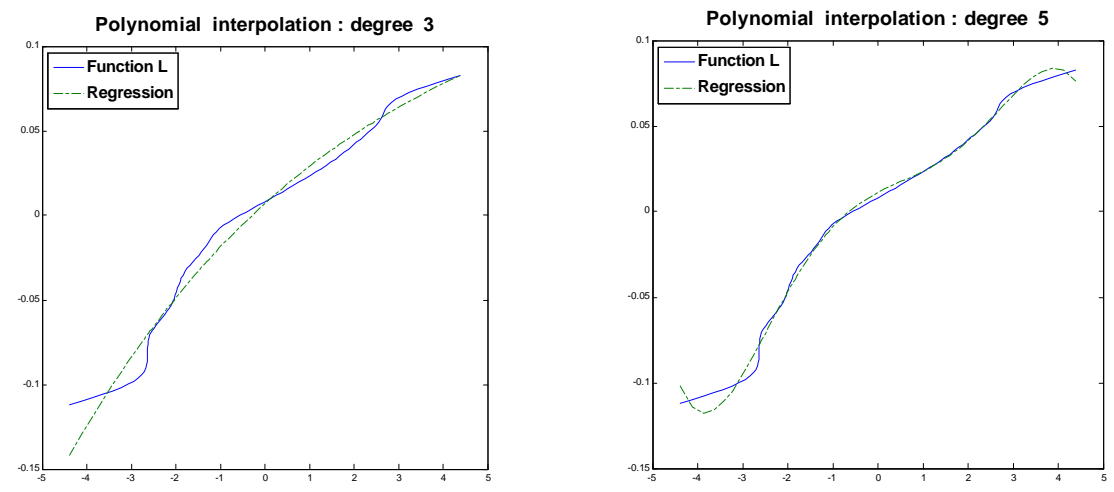

Figure 7: Polynomial interpolation of function 1 of the Multi Strategy index 


\subsection{Comparaison between optimal payoffs}

The second part of the numerical illustration is to compare between the cumulative distribution functions (CDF) of optimal payoff of tow insured portfolios in the presence of a CRRA utility function (denote $\alpha=1-\gamma$ ). For the first portfolio we assume that the risky asset follows Johnson $S_{U}$ distribution and for the second portfolio, we assume that the risky asset follows a Gaussian distribution. We have specifically chose the function $S_{U}$ since as we have found in the foregoing, most of the risky assets returns (the hedge fund index returns in our study), follow this distribution.

By assuming that the function $g($.$) is of the type g(y)=\ln \left(y+\sqrt{1+y^{2}}\right)=$ $\sinh ^{-1}(y)$, the process $\beta_{t}$ of equation (42) has the following form:

$$
\begin{aligned}
& g^{\prime}(y)=\frac{1}{\sqrt{y^{2}+1}} \\
& g^{\prime \prime}(y)=-\frac{y}{\left(y^{2}+1\right)^{\frac{3}{2}}} \\
& \beta_{t}=\frac{\delta}{\lambda} r \frac{1}{\sqrt{y^{2}+1}}-\frac{1}{2 \delta} \frac{y}{y^{2}+1}-\frac{\lambda}{2 \delta} \sqrt{y^{2}+1}
\end{aligned}
$$

The following figures illustrate both return cumulative distributions and payoffs corresponding to optimal uninsured and insured portfolios for the multi strategy index. For this purpose, we consider the following values for parameters: $T=1 ; p=0.9 ; \alpha=1-\gamma=0.1$.

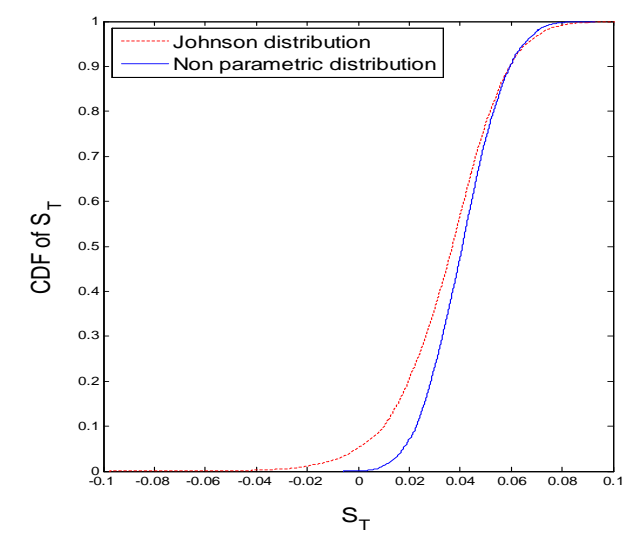

Figure 8: Comparaison between ST of Johnson distribution and ST of Non parametric distribution

Figure (8) provides both the return cumulative distribution functions of the risky asset (here, the multi strategy index), namely the cdf estimated from the 
Johnson assumption and the generalized Johnson cdf ("non parametric distribution"). For this empirical example, we note that the Johnson cdf is stochastically dominated at the second-order by the non parametric cdf, which implies that the Johnson cdf is more "conservative' that the generalized Johnson cdf. For example, for most values $s$ of $S_{T}$, the Johnson cdf provides smaller probabilities to be above $s$.

One important factor is the process $\beta$ that determines the pricing (as emphasized in previous section). Figure (9) shows that the two processes $\beta$ are quite different. For the Johnson distribution, the process $\beta$ is increasing while, for the generalized Johnson distribution, it is first increasing then decreasing.
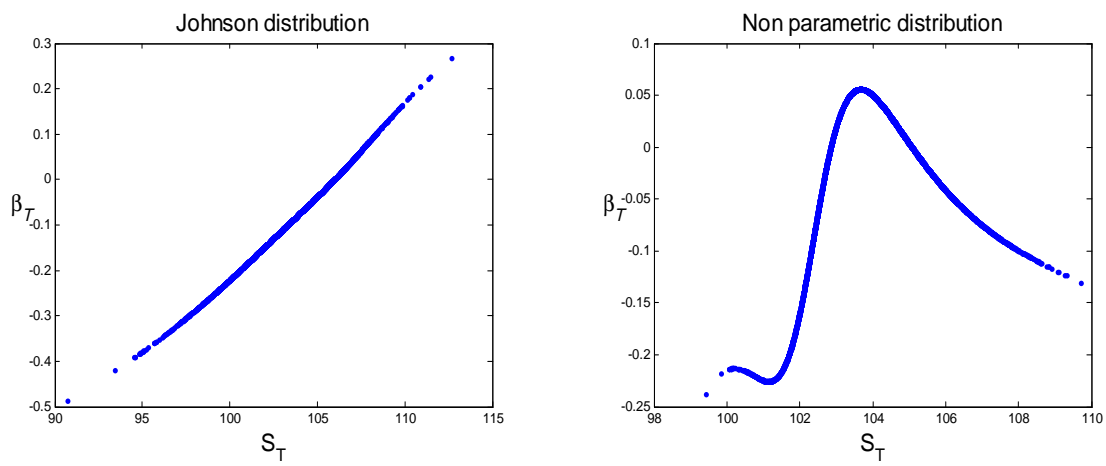

Figure 9: Values of Beta

Figure (10) displays the two corresponding payoffs. As expected from previous features of process $\beta$, the first payoff is increasing while, for the generalized Johnson distribution, it is not monotone.
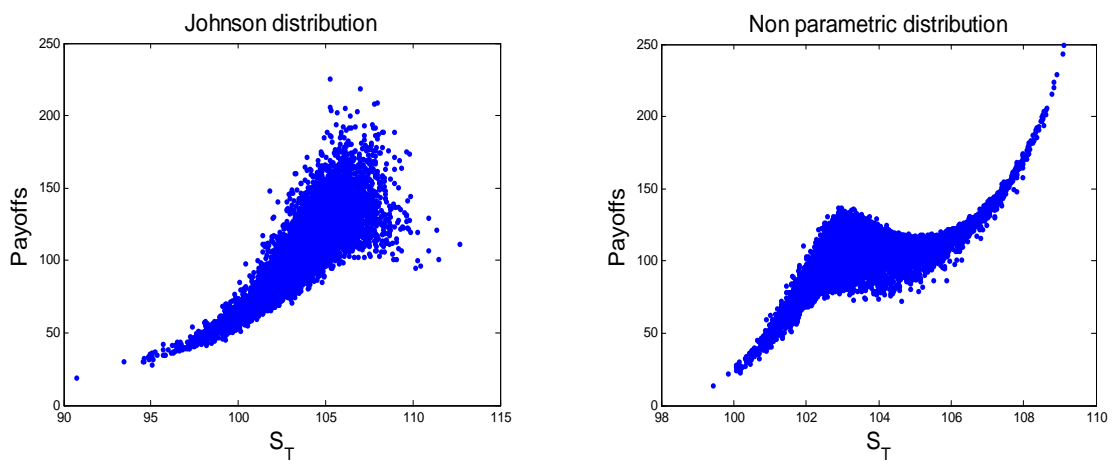

Figure 10: Payoffs of uninsured protfolios 
Figure (11) displays the two corresponding portfolio return cdfs.
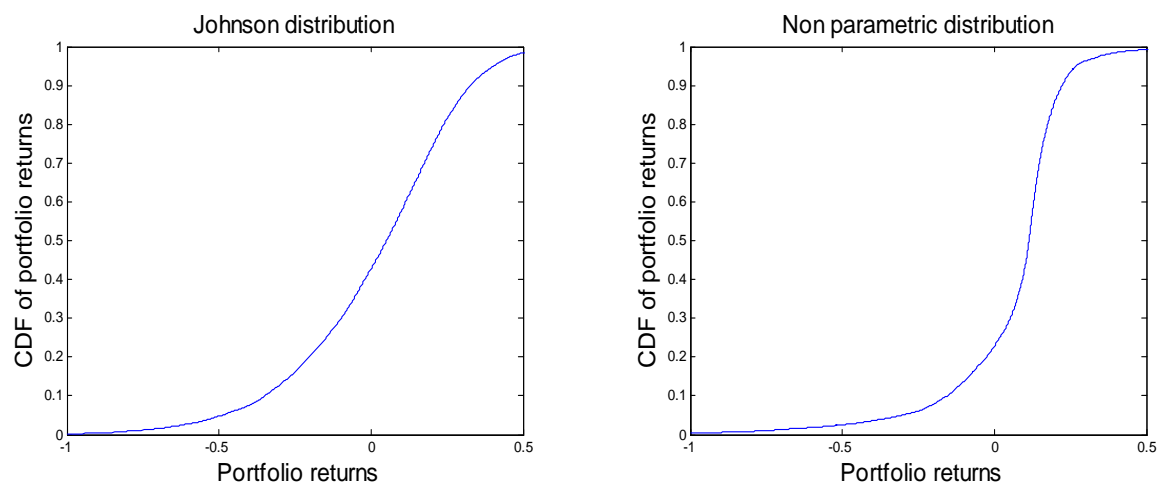

Figure 11: CDF of uninsured protfolios returs

Now we consider portfolio with insurance constraints. Figure (12) displays the two corresponding payoffs. As expected from previous results for non insured portfolios, the first payoff is increasing while, for the generalized Johnson distribution, it is not monotone.
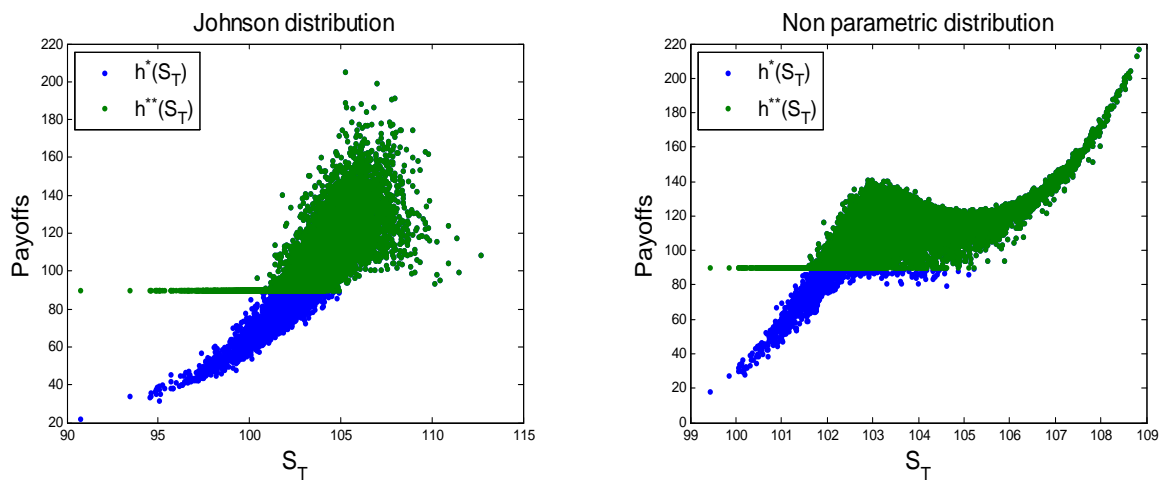

Figure 12: Payoffs of insured protfolios 
Figure (13) displays the two corresponding portfolio return cdfs for the insurance case.
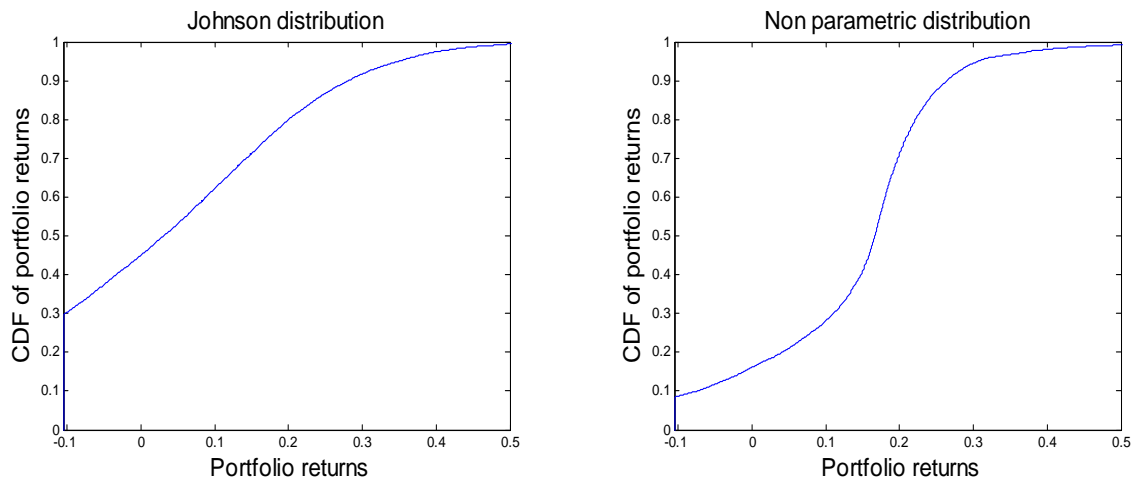

Figure 13: CDF of insured protfolios returs

To better compare the two cdfs, we plot them simultaneously in Figure (14). As for the multi strategy index itself, we note that the Johnson portfolio return cdf is stochastically dominated at the second-order by the generalized Johson cdf, which implies that the Johnson cdf is more "conservative' that the generalized Johnson cdf. This is due to the fact that the standard Johnson cdf is too "optimistic" about multi strategy index performances, while the generalized Johnson distribution (which better fits actual data) allows to take more account of risk.
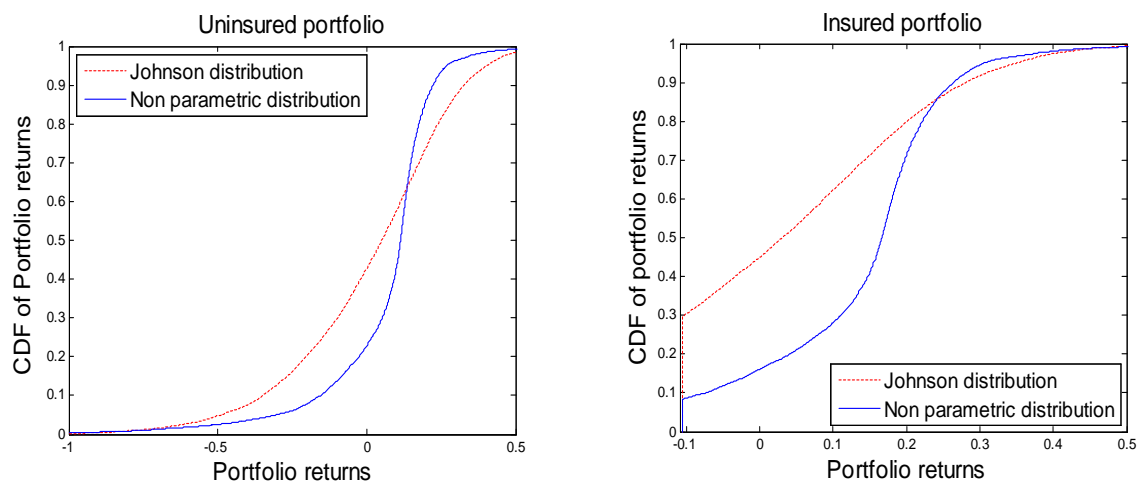

Figure 14: Comparaison between CDF of portfolio with Johnson distribution and CDF of of portfolio with Non parametric distribution 


\section{Conclusion}

By using the expected utility criterion, we can determine the optimal portfolio payoff in the case of a generalized distribution of Johnson, with or without portfolio insurance constraint. The optimal portfolio payoff function at maturity depends clearly on the investor risk aversion and on the requested guarantee. In the case of a power utility function (CRRA case), and when the dynamics of the risky asset is a geometric Brownian motion, the value of the uninsured optimal portfolio is a power function of the reference risky asset value. The concavity / convexity of the optimal payoff function is determined by the Sharpe ratio (that represents the financial market performance) divided by the relative risk aversion (which models the investor risk attitude). In the case of Johnson distributions and of a CRRA utility function, we prove that the value of the uninsured optimal portfolio is also a power function of a process taking the whole of risky asset trajectory into account. In all cases, the introduction of a guarantee at maturity implies the following general form: "guaranteed capital + call defined on an optimal portfolio without guarantee". Computation of higher moments confirms that Johnson distributions can better model the relatively extreme risks of financial assets. 


\section{References}

[1] Amenc, N., Curtis, S. and Martellini, L., The alpha and omega of hedge fund performance measurement. Working Paper, EDHEC Risk and Asset Management Research Center, 2003.

[2] Aitchison, J. and Brown, J.A.C., The lognormal distribution. Cambridge University Press, 1957.

[3] Basak, S., A general equilibrium model of portfolio insurance. Review of Financial studies, 1995, 8 1059-1090.

[4] Bertrand, P., Lesne, J-P. and Prigent, J-L., Gestion de portefeuille avec garantie : l'allocation optimale en actifs dérivés. Finance, 2001, 22 7-35.

[5] Bertrand, P. and Prigent, J-L. Gestion de Portefeuille: Analyse Quantitative et Gestion Structurée. Economica (Paris), 2006.

[6] Buhlmann, H., Delbaen, F. and Embrechts, P., No-Arbitrage, Change of Measure and Conditional Esscher Transforms. CWI Quarterly, 1996, 9 2913.

[7] Black, F. and Jones, R., Simplifying portfolio insurance. Journal of Portfolio Management, 1987, 14 48-51.

[8] Black, F., and Rouhani, R., Constant proportion portfolio insurance and the synthetic put option : a comparison, in Institutional Investor focus on Investment Management. edited by Frank J. Fabozzi., Cambridge, Mass. : Ballinger, 695-708,1989.

[9] Black, F. and Perold, A.R., Theory of constant proportion portfolio insurance. Journal of Economics, Dynamics and Control, 1992, 16 403-426.

[10] Bookstaber, R. and Langsam, J.A., Portfolio insurance trading rules. Journal of Futures Markets, 2000, 8 15-31.

[11] Brennan, M.J. et Solanki, R., Optimal portfolio insurance. Journal of Financial and Quantitative Analysis, 1981, 16 279-300.

[12] Breeden, D. and Litzenberger, R., Prices of state contingent claims implicit in options prices. Journal of Business, 1978, 51 621-651.

[13] Brooks, C. and Kat, H. M. The statistical properties of hedge fund index returns and their implications for investors. Journal of Alternative Investments, 2002, 5 26-44.

[14] Carr, P. and Madan, D., Optimal positioning in derivative securities. Quantitative Finance,2001, 1 9-37.

[15] Cox, J. and Huang, C.F. Optimal consumption and portfolio policies when assets prices follow a diffusion process. Journal of Economic Theory, 1989, 49 33-83. 
[16] Cvitanic, J. and Karatzas, I. Contingent claim valuation and hedging with constrained portfolio. IMA volume in Math, Davis M., Duffie D., Fleming, W. and Shreve, S. eds ,65, 13-33, 1996.

[17] Ekeland, I. and Turnbull, T., Infinite-Dimensional Optimization and Convexity. Chicago lectures in Mathematics, the University of Chicago Press, 1983.

[18] El Karoui, N., Jeanblanc, M. and Lacoste, V., Optimal portfolio management with American capital guarantee. Journal of Economics Dynamics and Control, 2001, 21 1377-1403.

[19] Fung, W. and Hsieh, D.A., Empirical characteristics of dynamic trading strategies : the case of hedge funds. Review of Financial Studies, 2001, 10 275-302.

[20] Grossman, S. and Vila, J.L., Portfolio insurance in complete markets: a note. Journal of Business, 1989, 60 275-298.

[21] Grossman, S. and Zhou, J., Equilibrium analysis of portfolio insurance, Journal of Finance, 1996, 51 1379-1403.

[22] Harrison J. M. and Kreps, D. Martingales and Arbitrage in Multiperiod Securities Markets, Journal of Economic Theory, 1979, 20 381-408.

[23] Harrison J. M. and Pliska, S.R., Martingales and Stochastic Integrals in the Theory of Continuous Trading », Stochastic Processes and their Applications, 1981, 11 215-260.

[24] Hill, I.D., Hill, R., and Holder, R.L., Fitting Johnson curves by moments. Applied Statistics, 1976, AS99.

[25] Jacod, J., and Shiryaev, A.N., Limit Theorems for Stochastic Processes. Berlin: Springer, 2003.

[26] Jensen, B.A. and Sorensen, C., Paying for minimum interest rate guarantees: who should compensate who ?. European Financial Management, 2001, 7 183-211.

[27] Johnson, N.L., Systems of frequency curves generated by methods of translation. Biometrica, 1949, 58 547-558.

[28] Karatzas, I., Lehoczky, J., Sethi, S.P. and Shreve, S.E., Explicit solution of a general consumption/investment problem. Mathematics of Operations Research, 1986, 11 261-294.

[29] Leland, H.E. and Rubinstein, M.,The evolution of portfolio insurance. in: D.L. Luskin, ed., Portfolio insurance: a guide to dynamic hedging, Wiley, 1976. 
[30] Leland, H., Who should buy portfolio insurance ?. Journal of Finance, 1980, 35 581-594.

[31] Leland, H.E. and Rubinstein, M., The evolution of portfolio insurance. in: D.L. Luskin, ed., Portfolio insurance: a guide to dynamic hedging, Wiley, 1991.

[32] Passow, A., Omega Portfolio Construction with Johnson Distributions, FAME Research Paper N 120, HEC Geneva, 2004.

[33] Perold, A., Constant portfolio insurance. Harvard Business School. Unpublished manuscript, 1986.

[34] Perold, A. and Sharpe, W., Dynamic strategies for asset allocation. Financial Analyst Journal,1998, 51 16-27.

[35] Prigent, J-L., Assurance du portefeuille : analyse et extension de la méthode du coussin. Bankers, Markets and Investors, 2001, 51 33-39.

[36] Prigent, J-L., Generalized option based portfolio insurance. Working Paper THEMA, University of Cergy-Pontoise, France, 2006.

[37] Prigent, J.-L., Portfolio Optimization and Performance Analysis. Boca Raton (Florida): Chapman \& Hall, 2007.

[38] Slifker, J.F. and Shapiro, S.S., The Johnson system: selection and parameter estimation. Technometrics, 1980, 22 239-246.

[39] Wheeler, Robert E., Quantile estimators of Johnson curve parameters. Biometrika, 1980, 67 725-728. 


\section{Appendices}

\section{Appendix A (Optimal payoff in presence of guarantee)}

The proof is similar to that of El Karoui et al. (2001). Consider the solution $V_{T}^{*}$ the problem without constraint guarantee. By using Cox and Huang (1989), the solution is given by:

$$
V_{T}^{e}=J\left(\rho M_{T}\right),
$$

where the Lagrange parameter $\rho$ is such that $V_{0}^{e}=\mathbb{E}_{\mathbb{P}}\left[V_{T}^{e} M_{T}\right]$.

On the other hand, for each portfolio $V_{T}$ with an initial investment $V_{0}$ satisfying $V_{T} \geq F_{T}$, that the utility $U$ is concave, we have:

$$
U\left(V_{T}\right)-U\left(V_{T}^{*}\right) \leq U^{\prime}\left(V_{T}^{*}\right)\left(V_{T}-V_{T}^{*}\right) .
$$

Since $U^{\prime}$ is increasing, we deduce :

$$
U^{\prime}\left(V_{T}^{*}\right)\left(V_{T}-V_{T}^{*}\right)=\operatorname{Min}\left(\rho M_{T}, U^{\prime}\left(F_{T}\right)\right)\left(V_{T}-V_{T}^{*}\right) .
$$

Furthermore, we have:

$$
\operatorname{Min}\left(\rho M_{T}, U^{\prime}\left(F_{T}\right)\right)\left(V_{T}-V_{T}^{*}\right)=\rho M_{T}\left(V_{T}-V_{T}^{*}\right)-\left[\rho M_{T}-U^{\prime}\left(F_{T}\right)\right]^{+}\left(V_{T}-F_{T}\right) .
$$

Finally, the fact that $E_{P}\left[V_{T} M_{T}\right]=V_{0}^{*}=E_{P}\left[V_{T}^{*} M_{T}\right]$, we obtain:

$$
\mathbb{E}_{\mathbb{P}}\left[\operatorname{Min}\left(\rho M_{T}, U^{\prime}\left(F_{T}\right)\right)\left(V_{T}-V_{T}^{*}\right)\right]=-\mathbb{E}_{\mathbb{P}}\left[\left[\rho M_{T}-U^{\prime}\left(F_{T}\right)\right]^{+}\left(V_{T}-F_{T}\right)\right] \leq 0 .
$$

Consequently, we deduce:

$$
\mathbb{E}_{\mathbb{P}}\left[U\left(V_{T}\right)\right] \leq \mathbb{E}_{\mathbb{P}}\left[U\left(V_{T}^{*}\right)\right]
$$

\section{Appendix B (Optimal payoff as function of the risky asset path)}

In what follows, we detail the path dependence of the optimal portfolio within Johnson processes (proof of Corollary 19).

Recall that the optimal portfolio value is given by:

$$
V_{T}^{*}=J\left(\rho \eta_{T}\right),
$$

with

$$
\eta_{t}=\eta_{0} \mathcal{E}\left[\tau_{t}\right]=\eta_{0} \exp \left[-\frac{1}{2} \int_{0}^{t} \beta_{s}^{2} d s+\int_{0}^{t} \beta_{s} d W_{s}\right]
$$

and:

$$
\beta_{t}=-\frac{-r+d A_{t}^{X} / d t+1 / 2 \lambda_{t}^{2}\left[1 / g^{\prime}\left(\frac{\ln \left(\frac{S_{t}}{S_{0}}\right)-\xi_{t}}{\lambda_{t}}\right)\right]^{2} \frac{1}{\delta_{t}^{2}}}{\lambda_{t} 1 / g^{\prime}\left(\frac{\ln \left(\frac{S_{t}}{S_{0}}\right)-\xi_{t}}{\lambda_{t}}\right) \frac{1}{\delta_{t}}},
$$


First step: (analysis of process $\beta$ )

Then, from Relations (6) and (7), we deduce:

$$
\begin{gathered}
d A_{t}^{X} / d t=\lambda_{t}^{\prime}\left(\frac{\ln \left(\frac{S_{t}}{S_{0}}\right)-\xi_{t}}{\lambda_{t}}\right)+ \\
+\lambda_{t}\left[1 / g^{\prime}\left(\frac{\ln \left(\frac{S_{t}}{S_{0}}\right)-\xi_{t}}{\lambda_{t}}\right)\right]\left(-\frac{\delta_{t}^{\prime}}{\delta_{t}^{2}}\left(\delta_{t} g\left(\frac{\ln \left(\frac{S_{t}}{S_{0}}\right)-\xi_{t}}{\lambda_{t}}\right)+\gamma_{t}\right)-\frac{\gamma_{t}^{\prime} \delta_{t}-\gamma_{t} \delta_{t}^{\prime}}{\delta_{t}^{2}}\right) \\
-\frac{\lambda_{t}}{2 \delta_{t}^{2}}\left(\frac{g^{\prime \prime}}{g^{\prime 3}}\right)\left(\frac{\ln \left(\frac{S_{t}}{S_{0}}\right)-\xi_{t}}{\lambda_{t}}\right)+\xi_{t}^{\prime} .
\end{gathered}
$$

Consequently, $d A_{t}^{X} / d t$ is a deterministic function of $S_{t}$ and so does process $\beta$.

Second step: (analysis of process $W$ as function of $S$ )

Recall that:

$$
W_{t}=\gamma_{t}+\delta_{t} g\left(\frac{\ln \left(\frac{S_{t}}{S_{0}}\right)-\xi_{t}}{\lambda_{t}}\right) .
$$

Thus, at any time, we have:

$$
\frac{W_{t}-\gamma_{t}}{\delta_{t}}=g\left(\frac{\ln \left(\frac{S_{t}}{S_{0}}\right)-\xi_{t}}{\lambda_{t}}\right) .
$$

Applying standard stochastic calculus rules, we deduce:

$$
\begin{gathered}
d\left(\frac{\ln \left(\frac{S_{t}}{S_{0}}\right)-\xi_{t}}{\lambda_{t}}\right)= \\
-\frac{\lambda_{t}^{\prime}}{\lambda_{t}^{2}} \ln \left(\frac{S_{t}}{S_{0}}\right) d t+\frac{d X_{t}+1 / 2 d\langle X, X\rangle_{t}}{\lambda_{t}}-\frac{\xi_{t}^{\prime} \lambda_{t}-\xi_{t} \lambda_{t}^{\prime}}{\lambda_{t}^{2}} d t .
\end{gathered}
$$

Therefore, we get:

$$
\begin{aligned}
d W_{t}= & \gamma_{t}^{\prime} d t+\delta_{t}^{\prime} g\left(\frac{\ln \left(\frac{S_{t}}{S_{0}}\right)-\xi_{t}}{\lambda_{t}}\right) d t \\
& +\delta_{t} g^{\prime}\left(\frac{\ln \left(\frac{S_{t}}{S_{0}}\right)-\xi_{t}}{\lambda_{t}}\right) d\left(\frac{\ln \left(\frac{S_{t}}{S_{0}}\right)-\xi_{t}}{\lambda_{t}}\right) \\
& +\frac{1}{2} \delta_{t} g^{\prime \prime}\left(\frac{\ln \left(\frac{S_{t}}{S_{0}}\right)-\xi_{t}}{\lambda_{t}}\right) \frac{d\langle X, X\rangle_{t}}{\lambda_{t}^{2}} .
\end{aligned}
$$


Note that

$$
d X_{t}+1 / 2 d\langle X, X\rangle_{t}=d S_{t} / S_{t}
$$

and

$$
d\langle X, X\rangle_{t}=\lambda_{t}^{2}\left[1 / g^{\prime}\left(\frac{\ln \left(\frac{S_{t}}{S_{0}}\right)-\xi_{t}}{\lambda_{t}}\right)\right]^{2} \frac{1}{\delta_{t}^{2}} d t .
$$

Finally, we have:

$$
\begin{gathered}
d W_{t}=\gamma_{t}^{\prime} d t+\delta_{t}^{\prime} g\left(\frac{\ln \left(\frac{S_{t}}{S_{0}}\right)-\xi_{t}}{\lambda_{t}}\right) d t \\
+\delta_{t} g^{\prime}\left(\frac{\ln \left(\frac{S_{t}}{S_{0}}\right)-\xi_{t}}{\lambda_{t}}\right) \times \\
\left(-\frac{\lambda_{t}^{\prime}}{\lambda_{t}^{2}} \ln \left(\frac{S_{t}}{S_{0}}\right) d t+\frac{d S_{t}}{\lambda_{t} S_{t}}-\frac{\xi_{t}^{\prime} \lambda_{t}-\xi_{t} \lambda_{t}^{\prime}}{\lambda_{t}^{2}} d t\right) \\
+\frac{1}{2 \delta_{t}} \frac{g^{\prime \prime}\left(\frac{\ln \left(\frac{S_{t}}{S_{0}}\right)-\xi_{t}}{\lambda_{t}}\right)}{\left[g^{\prime}\left(\frac{\ln \left(\frac{S_{t}}{S_{0}}\right)-\xi_{t}}{\lambda_{t}}\right)\right]^{2}} d t .
\end{gathered}
$$

Thus we note that $d W_{t}$ has the following form:

$$
d W_{t}=\widehat{a}\left(t, S_{t}\right) d t+\widehat{b}\left(t, S_{t}\right) d S_{t},
$$

where $\widehat{a}(t,$.$) and \widehat{b}(t,$.$) are deterministic functions.$ 